\title{
How planetary growth outperforms migration
}

\author{
Anders Johansen ${ }^{1}$, Shigeru $\operatorname{Ida}^{2}$, and Ramon Brasser ${ }^{2}$ \\ ${ }^{1}$ Lund Observatory, Department of Astronomy and Theoretical Physics, Lund University, Box 43, 22100 Lund, Sweden \\ e-mail: anders@astro.lu.se \\ ${ }^{2}$ Earth-Life Science Institute (ELSI), Tokyo Institute of Technology, Meguro, Tokyo 152-8550, Japan
}

Received 11 August 2018 / Accepted 10 January 2019

\begin{abstract}
Planetary migration is a major challenge for planet-formation theories. The speed of type-I migration is proportional to the mass of a protoplanet, while the final decade of growth of a pebble-accreting planetary core takes place at a rate that scales with the mass to the two-thirds power. This results in planetary growth tracks (i.e., the evolution of the mass of a protoplanet versus its distance from the star) that become increasingly horizontal (migration dominated) with the rising mass of the protoplanet. It has been shown recently that the migration torque on a protoplanet is reduced proportional to the relative height of the gas gap carved by the growing planet. Here we show from 1D simulations of planet-disc interaction that the mass at which a planet carves a $50 \%$ gap is approximately 2.3 times the pebble isolation mass. Our measurements of the pebble isolation mass from 1D simulations match published 3D results relatively well, except at very low viscosities $\left(\alpha<10^{-3}\right)$ where the 3D pebble isolation mass is significantly higher, possibly due to gap edge instabilities that are not captured in 1D. The pebble isolation mass demarks the transition from pebble accretion to gas accretion. Gas accretion to form gas-giant planets therefore takes place over a few astronomical units of migration after reaching first the pebble isolation mass and, shortly after, the $50 \%$ gap mass. Our results demonstrate how planetary growth can outperform migration both during core accretion and during gas accretion, even when the Stokes number of the pebbles is small, $S t \sim 0.01$, and the pebble-to-gas flux ratio in the protoplanetary disc is in the nominal range of $0.01-0.02$. We find that planetary growth is very rapid in the first million years of the protoplanetary disc and that the probability for forming gas-giant planets increases with the initial size of the protoplanetary disc and with decreasing turbulent diffusion.
\end{abstract}

Key words. planet-disk interactions - planets and satellites: formation - planets and satellites: gaseous planets

\section{Introduction}

The formation of planets involves many distinct steps in the growth from dust and ice particles to a full planetary system (a realisation that was pioneered 50 years ago by Safronov 1969). The competition between radial migration and growth is nevertheless a general theme in planet formation. The formation of planetesimals, for example, has to overcome the radial drift caused by the head-wind of the slower-moving gas on the growing particles (Weidenschilling 1977; Brauer et al. 2008). Trapping pebbles in pressure bumps formed in the turbulent gas flow (Lyra et al. 2008; Johansen et al. 2009a; Bai 2015), or by embedded planets (Lyra et al. 2009), or through run-away pileups caused by the friction on the gas (Youdin \& Goodman 2005; Johansen et al. 2009b; Bai \& Stone 2010; Drążkowska et al. 2016; Gonzalez et al. 2017) provide possible solutions to the radial drift problem of planetesimal formation.

Planetary migration results from the gravitational torque exerted on a protoplanet by the spiral density wakes excited in the gaseous protoplanetary disc (Lin \& Papaloizou 1979; Goldreich \& Tremaine 1980; Tanaka et al. 2002). The nominal type-I migration rate reaches approximately $1 \mathrm{~m} \mathrm{~s}^{-1}$ (about $100 \mathrm{AU} \mathrm{Myr}^{-1}$ ) for a protoplanet of ten earth masses embedded in a young, massive protoplanetary disc. The solutions proposed to mitigate catastrophic type-I migration include slowing down or even reversing the migration where the temperature gradient is steep in the inner regions of the protoplanetary disc (Paardekooper et al. 2010; Lyra et al. 2010; Bitsch et al. 2014; Brasser et al. 2017), or by a positive corotation torque resulting from the accretion heat of the protoplanet (Benítez-Llambay et al. 2015) or from the scattering of (large) pebbles (Benítez-Llambay \& Pessah 2018). Migration can even stall entirely if the turbulent viscosity is too weak to diffuse away the gas density enhancement that forms inside the planetary orbit (Rafikov 2002; Li et al. 2009; Fung \& Lee 2018).

A more direct way to overcome type-I migration is simply for the planetary core to increase its mass very rapidly. The accretion rate of pebbles is potentially high enough for the protoplanet growth to outperform the nominal rate of type-I migration (Lambrechts \& Johansen 2014; Ormel et al. 2017; Johansen \& Lambrechts 2017). Bitsch et al. (2015a) demonstrated that protoplanets forming several tens of astronomical units from the host star have enough space to grow to gas-giant planets while they migrate into orbits ${ }^{1}$ of 5-10 AU. However, Brügger et al. (2018) found that the pebble flux rate in the model of Bitsch et al. (2015a) was artificially high and that nominal pebble fluxes $(\sim 0.01$ relative to the gas flux) do not yield high-enough core growth rates to compete with migration, unless the metallicity is enhanced significantly beyond the solar value. Bitsch et al. (2018a) argued instead that the pebble sizes and surface densities of Bitsch et al. (2015a) in fact correspond well to observations of protoplanetary discs. Nevertheless, although the protoplanetary disc model advocated in Bitsch et al. (2018a) is motivated observationally, the pebble sizes and surface densities used in

\footnotetext{
1 Matsumura et al. (2017), on the other hand, started the seeds in the 5-10 AU region and showed how this leads mainly to the formation of
} warm and hot Jupiters. 
that work are not anchored in self-consistent theoretical models of pebble growth and drift.

In this paper we develop pebble-accretion models with small pebbles of Stokes number St $\sim 0.01$ (approximately mm-sized in the planet-formation region), much smaller than those considered in Bitsch et al. (2015a) and Brügger et al. (2018). Such small pebbles follow the viscous accretion flow of the gas and, in contrast to larger pebbles, their column density is not notably reduced by radial drift (Lambrechts \& Johansen 2014). We demonstrate how in this model planetary formation outperforms migration for nominal pebble fluxes and metallicities. We derive analytical expressions that describe the growth tracks of solid protoplanets undergoing pebble accretion (Sect. 2). We find that planetary cores do undergo substantial migration before reaching the pebble isolation mass. However, using novel prescriptions for the migration rate of gap-opening planets (Kanagawa et al. 2018), we show that gas accretion to form gas-giant planets takes place over just a few astronomical units of migration (Sect. 3). Hence the main migration phase of a protoplanet happens during the accumulation of the core. We summarise our results and discuss the implications for planet formation in Sect. 4. In Appendix A we discuss the survival of pebbles in protoplanetary discs and in Appendix B we derive approximate times associated with the analytical growth tracks from Sect. 2. Our paper forms a companion paper to Ida et al. (2018), which focuses on the effect of the Kanagawa et al. (2018) migration rate on the gas accretion stage.

\section{Analytical pebble accretion growth tracks}

In this section we derive analytical expressions for the growth track of a protoplanet growing by pebble accretion. We then use this expression to derive the location where the growth track crosses the pebble isolation mass.

\subsection{Pebble accretion}

To derive the analytical shape of the growth track of a solid protoplanet, we use the protoplanet growth rate from pebble accretion in the 2D Hill regime (Ormel \& Klahr 2010; Lambrechts \& Johansen 2012; Ida et al. 2016),

$\dot{M}=2\left(\frac{S t}{0.1}\right)^{2 / 3} \Omega R_{\mathrm{H}}^{2} \Sigma_{\mathrm{p}}$.

Here $M$ is the mass of the protoplanet, $S t=\Omega \tau_{\mathrm{f}}$ is the Stokes number of the pebbles (defined later in Eq. (16)), $\Omega$ is the Keplerian frequency at the location of the protoplanet, $\tau_{\mathrm{f}}$ is the friction time of the pebble (proportional to the particle size when pebbles are small), $R_{\mathrm{H}}=\left[M /\left(3 M_{\star}\right)\right]^{1 / 3} r$ is the Hill radius of the protoplanet, $M_{\star}$ is the mass of the central star, $r$ is the radial location of the planet and $\Sigma_{\mathrm{p}}$ is the pebble surface density. Significant effort is currently being put into understanding how pebble accretion depends on the eccentricity and inclination of the protoplanet (Johansen et al. 2015; Liu \& Ormel 2018) as well as on realistic hydrodynamical flow in the Hill sphere (Popovas et al. 2018), but we consider here the more simple case of a protoplanet on a circular orbit and the gas flow as pure shear.

Our assumption of $2 \mathrm{D}$ Hill accretion is valid when the pebble-accretion radius, $R_{\text {acc }}=(S t / 0.1)^{1 / 3} R_{\mathrm{H}}$, is larger than the scale height of the pebble layer, $H_{\mathrm{p}}=H \sqrt{\delta / S t}$ (see Morbidelli et al. 2015, for a discussion). Here $H$ is the scale-height of the gas and $\delta$ is the dimensionless dust diffusion coefficient (defined in Johansen et al. 2014). This gives an accretion radius relative to the pebble scale height as

$\frac{R_{\mathrm{acc}}}{H_{\mathrm{p}}}=0.9\left(\frac{S t}{0.01}\right)^{1 / 3}\left(\frac{M}{M_{\mathrm{E}}}\right)^{1 / 3}\left(\frac{H / r}{0.05}\right)^{-1}\left(\frac{S t / \delta}{100}\right)^{1 / 2}$

We normalised here to $S t=0.01$ and $\delta=0.0001$. Our choice of Stokes number $S t=0.01$ is elaborated in Sect. 2.4, while the choice of a low diffusion coefficient $\delta$ is motivated by observations of dusty protoplanetary discs that show that the dust has settled to a mid-plane layer with a width equal to $10 \%$ of a gas scale height (Mulders \& Dominik 2012; Menu et al. 2014; Pinte et al. 2016). We note that $S t / \delta=100$ indeed yields a moderately sedimented pebble mid-plane layer with a scale height relative to the gas scale height of $H_{\mathrm{p}} / H=0.1$.

The transition from the Bondi regime (where the pebble approach speed is set by the sub-Keplerian motion) to the Hill regime (where the pebble approach speed is set by the Keplerian shear) of pebble accretion typically happens at 0.001-0.01 Earth masses (Lambrechts \& Johansen 2012). Protoplanets that accrete either in the Bondi regime or in the 3D Hill regime thus experience very low migration rates and hence the shape of the growth track is not affected by our choice to start the analytical core growth tracks in the 2D Hill accretion phase. In Sect. 3, we include the 3D branch of Hill accretion in the numerical integrations.

Johansen \& Lambrechts (2017) demonstrated that the accretion of planetesimals contributes significantly to the growth from planetesimal sizes to protoplanets with masses of $0.001-0.01 M_{\mathrm{E}}$, due to the low rates of pebble accretion in the Bondi regime, while pebble accretion in the Hill regime dominates the further growth. Therefore, we start our protoplanets at $M_{0}=0.01 M_{\mathrm{E}}$ and ignore the contribution of planetesimal accretion to the core growth rate, although the inclusion of planetesimal accretion could give a boost to the accretion rate, depending on the migration speed of the protoplanet (Tanaka \& Ida 1999).

\subsection{Type-I migration}

For type-I migration we use the standard scaling law,

$\dot{r}=-k_{\mathrm{mig}} \frac{M}{M_{\star}} \frac{\Sigma_{\mathrm{g}} r^{2}}{M_{\star}}\left(\frac{H}{r}\right)^{-2} v_{\mathrm{K}}$.

Here $\dot{r}$ is the migration speed of the protoplanet, $k_{\mathrm{mig}}$ is a constant prefactor that depends on the gradients of surface density and temperature, $M_{\star}$ is the mass of the central star, $\Sigma_{\mathrm{g}}$ is the gas surface density, $H / r$ is the disc aspect ratio and $v_{\mathrm{K}}$ is the Keplerian speed at the position of the planet. For the prefactor $k_{\text {mig }}$ we follow here a fit to 3D numerical simulations found in D’Angelo \& Lubow (2010),

$k_{\text {mig }}=2(1.36+0.62 \beta+0.43 \zeta)$,

where $\beta$ and $\zeta$ are the negative logarithmic derivatives of the surface density and the temperature profiles, respectively. However, we explicitly maintain $k_{\text {mig }}$ in our equations, since the prefactor depends on the physical effects that are included in the simulations (e.g. Tanaka et al. 2002; Paardekooper et al. 2010).

\subsection{Radial mass fluxes of gas and pebbles}

The gas sound speed $c_{\mathrm{s}}$ and the derived gas scale-height $H=c_{\mathrm{S}} / \Omega$ enter both the calculation of the planetary migration rate as well as expressions for the radial speed of the gas and 
pebbles that we present below. We assume that the sound-speed profile follows a power law,

$c_{\mathrm{s}}=c_{\mathrm{s} 1}\left(\frac{r}{\mathrm{AU}}\right)^{-\zeta / 2}$.

Here $\zeta$ is the negative power-law index of the temperature (proportional to $c_{\mathrm{s}}^{2}$ ) and $c_{\mathrm{s} 1}$ is the sound speed at $1 \mathrm{AU}$. The disc aspect ratio then follows the power law

$\frac{H}{r} \propto r^{-\zeta / 2+1 / 2}$.

The turbulent viscosity $v$ sets the radial gas accretion speed. We use here the $\alpha$-disc assumption for the turbulent viscosity (e.g. Pringle 1981),

$v=\alpha c_{\mathrm{s}} H$.

This now results in the gas accretion speed

$u_{r}=-\frac{3}{2} \frac{v}{r}=-\frac{3}{2} \alpha c_{\mathrm{s}} \frac{H}{r}$.

This expression for the accretion speed is specific to the $\alpha$-disc assumption and would not be valid if the angular momentum loss was transported instead by disc winds (Bai \& Stone 2013). However, since the radial mass-accretion rate of the gas depends only on the speed of the gas, and not on the nature of the angular momentum transport, we can consider $\alpha$ in Eq. (8) as simply a dimensionless measure of the radial accretion speed. 1977)

The radial drift of the particles is given by (Weidenschilling

$v_{r}=-\frac{2 \Delta v}{S t+S t^{-1}}+\frac{u_{r}}{1+S t^{2}}$.

In the limit $S t \ll 1$, valid for pebbles smaller than approximately $0.1-1 \mathrm{~m}$ in size outside of a few astronomical units (Johansen et al. 2014), this expression simplifies to

$v_{r}=-2 S t \Delta v+u_{r}$.

Here the sub-Keplerian speed $\Delta v$, which is a measure of the radial pressure support of the gas, is given by

$\Delta v=-\frac{1}{2} \frac{H}{r}(\partial \ln P / \partial \ln r) c_{\mathrm{s}}$.

We denote the negative logarithmic pressure gradient in the mid-plane as $-\partial \ln P / \partial \ln r \equiv \chi=\beta+\zeta / 2+3 / 2$. The inwards mass fluxes of gas and pebbles, respectively, are given by

$\dot{M}_{\mathrm{g}}=-2 \pi r u_{r} \Sigma_{\mathrm{g}}$,

$\dot{M}_{\mathrm{p}}=-2 \pi r v_{r} \Sigma_{\mathrm{p}}$.

The ratio of the surface densities of pebbles to gas is then

$\frac{\Sigma_{\mathrm{p}}}{\Sigma_{\mathrm{g}}}=\frac{\dot{M}_{\mathrm{p}}}{\dot{M}_{\mathrm{g}}} \frac{u_{r}}{-2 S t \Delta v+u_{r}}=\frac{\xi}{(2 / 3)(S t / \alpha) \chi+1}$.

Here we defined the ratio of the fluxes as in Ida et al. (2016),

$\xi=\frac{\dot{M}_{\mathrm{p}}}{\dot{M}_{\mathrm{g}}}$.

We show below in Sects. 2.5 and 2.6 that $\xi$ is a key parameter that determines the shape of the growth track of a protoplanet and the total migration distance of the protoplanet before reaching the pebble isolation mass. The gas and solid components of the protoplanetary disc will accrete towards the star on the same timescale when $\xi \approx 0.01$, where 0.01 represents the metallicity $Z$ of the protoplanetary disc. This nominal value of $\xi$ is obtained when the radial drift of the pebbles is dominated by advection with the accreting gas, corresponding to $S t / \alpha \lesssim 1$ in Eq. (14). Therefore, also the local metallicity $\Sigma_{\mathrm{p}} / \Sigma_{\mathrm{g}}$ will keep its original value $Z$ for the nominal pebbles-to-gas flux ratio.

Large pebbles with $S t \gg \alpha$ in Eq. (14) experience an increase in $\xi$ proportional to $S t$ and hence the local metallicity $\Sigma_{\mathrm{p}} / \Sigma_{\mathrm{g}}$ is maintained at its global value $Z$. An increased Stokes number will nevertheless have an overall positive effect on the pebble accretion rate (through Eq. (1)), but such large pebbles are lost to radial drift on a shorter timescale than the gas accretion. This is the well-known radial drift problem of protoplanetary discs (Brauer et al. 2007). Another possibility is that $\xi$ is dictated by the production rate of pebbles in the outer regions of the protoplanetary disc (Lambrechts \& Johansen 2014; Bitsch et al. 2015a). In that case, $\xi$ is no longer directly coupled to $S t$ in Eq. (14) and the local metallicity $\Sigma_{\mathrm{p}} / \Sigma_{\mathrm{g}}$ falls proportional to the inverse Stokes number when $S t \gg \alpha$. Overall, there are then many advantages to considering small pebbles for pebble accretion models, in contrast to the large pebbles that were used in the models of Bitsch et al. (2015a) and Brügger et al. (2018).

\subsection{Pebble sizes}

Dust in protoplanetary discs grows to pebbles through coagulation (Brauer et al. 2007; Zsom et al. 2010). If particles stick perfectly when they collide, then growth continues until the radial drift timescale becomes comparable to the growth timescale, at Stokes numbers around $0.1-1$ in the region of giant planet formation (Birnstiel et al. 2012; Lambrechts \& Johansen 2014). Pebble accretion with such drift-limited pebble growth was explored in Bitsch et al. (2015b) and Brügger et al. (2018).

Here we focus instead on a different and perhaps more realistic mode of pebble growth where the pebbles experience bouncing or fragmenting collisions. Zsom et al. (2010) showed that the growth of silicate particles is limited to millimetre ( $\mathrm{mm}$ ) sizes by bouncing, based on extensive experimental data on collisions between silicate dust aggregates. Such experiments also show that collisions become fragmenting when the collision speed crosses a threshold value (Birnstiel et al. 2012).

Water ice, in contrast to silicates, has higher surface energy and is thus expected to experience growth beyond the bouncing barrier (Okuzumi et al. 2012), while $\mathrm{CO}_{2}$ ice (and likely $\mathrm{CO}$ ice as well) appears to have sticking properties similar to silicates (Musiolik et al. 2016a,b). The $\mathrm{CO}_{2}$ ice line sits at a temperature of approximately $70 \mathrm{~K}$ at solar abundances, corresponding to the 2-4 AU region in the late stages of protoplanetary disc evolution (Bitsch et al. 2015b; Madhusudhan et al. 2017). Hence, we expect that the growth of pebbles is limited by bouncing or fragmentation in the accretion region of the cores of cold gas giants. Ices of $\mathrm{CO}$ and $\mathrm{CO}_{2}$ under UV irradiation may, in turn, form longer refractory organic molecules (Muñoz Caro \& Schutte 2003). We ignore here the possibility that such organic molecules could be sticky and facilitate the formation of pebbles greater than millimetres in size (Lodders 2004).

Both pebble accretion (in the Hill regime) and radial drift depend on the Stokes number of the pebbles rather than on their physical sizes. The pebble Stokes number is calculated from the radius $R$ and material density $\rho_{\bullet}$ of the pebbles through 
the relation

$S t=\sqrt{2 \pi} \frac{R \rho_{\bullet}}{\Sigma_{\mathrm{g}}}=\sqrt{2 \pi} \frac{3 \pi v R \rho_{\bullet}}{\dot{M}_{\mathrm{g}}}$.

This yields the pebble size for a given value of $S t / \alpha$ as

$$
\begin{aligned}
R= & \frac{S t}{\alpha} \frac{\dot{M}_{\mathrm{g}}}{\sqrt{2 \pi} 3 \pi c_{\mathrm{s}} H \rho_{\bullet}} \\
= & 1.1 \mathrm{~mm}\left(\frac{S t}{\alpha}\right)\left(\frac{\dot{M}_{\mathrm{g}}}{10^{-8} M_{\odot} \mathrm{yr}^{-1}}\right)\left(\frac{c_{\mathrm{s} 1}}{6.5 \times 10^{2} \mathrm{~m} \mathrm{~s}^{-1}}\right)^{-2} \\
& \times\left(\frac{\rho_{\bullet}}{10^{3} \mathrm{~kg} \mathrm{~m}^{-3}}\right)^{-1}\left(\frac{r}{10 \mathrm{AU}}\right)^{\zeta-3 / 2} .
\end{aligned}
$$

In Sect. 3 we adopt a nominal protoplanetary disc evolution model where the gas-accretion rate drops from $10^{-7}$ to $10^{-8} M_{\odot} \mathrm{yr}^{-1}$ over $3 \mathrm{Myr}$ (Hartmann et al. 2016). This gives a typical pebble size of $\mathrm{mm}$ to centimetres $(\mathrm{cm})$ in size in the 5-20 AU region, for $S t / \alpha=1$, which corresponds well to the sizes of pebbles whose growth is stuck at the bouncing barrier (Zsom et al. 2010; Birnstiel et al. 2012).

The fragmentation barrier is reached when the collision speed driven by the turbulent gas motion, $v_{\mathrm{c}}=\sqrt{3 \alpha_{\mathrm{v}} S t} c_{\mathrm{s}}$ (Ormel \& Cuzzi 2007), equals an assumed fragmentation speed, $v_{\mathrm{f}}$ (Birnstiel et al. 2012). Here $\alpha_{\mathrm{v}}$ is the turbulent viscosity; we discuss its value and connection to the global disc accretion coefficient $\alpha$ in Sect. 2.6. The turbulent collision speed results in a limiting Stokes number

$$
\begin{aligned}
S t= & \frac{1}{3} \alpha_{\mathrm{v}}^{-1}\left(\frac{v_{\mathrm{f}}}{c_{\mathrm{s}}}\right)^{2}=0.003\left(\frac{\alpha_{\mathrm{v}}}{10^{-4}}\right)^{-1} \\
& \times\left(\frac{v_{\mathrm{f}}}{1 \mathrm{~m} \mathrm{~s}^{-1}}\right)^{2}\left(\frac{c_{\mathrm{s} 1}}{6.5 \times 10^{2} \mathrm{~m} \mathrm{~s}^{-1}}\right)^{-2}\left(\frac{r}{10 \mathrm{AU}}\right)^{\zeta} .
\end{aligned}
$$

The fragmentation barrier thus lies at a Stokes number that is (a) independent of the temporally decaying mass-accretion rate onto the star and (b) only weakly dependent on the distance from the star.

The small pebbles stuck at the bouncing or fragmentation barrier have radial speeds that are approximately the same as the radial gas accretion speed. This results in a pebble-to-gas flux ratio $\xi \approx Z$, where $Z$ is the overall metallicity of the protoplanetary disc, and hence a similar depletion time of the gaseous and solid components of the protoplanetary disc. For simplicity we therefore consider $S t \sim \alpha$ in our models, as this choice agrees both with the bouncing and fragmentation barriers and with observations that show that pebbles of $\mathrm{mm}-\mathrm{cm}$ sizes remain present in protoplanetary discs over a wide range of ages (Pérez et al. 2012; Huang et al. 2018). We discuss the lifetime of the pebble component of protoplanetary discs more in Appendix A.

Choosing a larger value of $S t / \alpha$ would correspond to larger pebbles, more in agreement with pebble growth limited only by the radial drift (Birnstiel et al. 2012; Lambrechts \& Johansen 2014). Such large pebbles would lead to high pebble-accretion rates, but would drain out of the protoplanetary disc on a shorter timescale than the gas-accretion timescale (Lin et al. 2018; our Appendix A), adding significant complication to modelling the divergent evolution of gas and pebbles.

\subsection{Analytical core growth track}

Using Eq. (1) for the core growth rate and Eq. (3) for the migration rate, we can now formulate the differential equation for the growth track $M(r)$,

$$
\begin{aligned}
\frac{\mathrm{d} M}{\mathrm{~d} r}= & \frac{\dot{M}}{\dot{r}} \\
= & -\frac{\xi}{(2 / 3)(S t / \alpha) \chi+1} \frac{2(S t / 0.1)^{2 / 3} M_{\star}\left(3 M_{\star}\right)^{-2 / 3}}{k_{\mathrm{mig}} G c_{\mathrm{s} 1}^{-2} \mathrm{AU}^{-\zeta}} \\
& \times r^{-\zeta} M^{-1 / 3} .
\end{aligned}
$$

The solution is found by separation of variables,

$$
\begin{aligned}
M^{4 / 3}-M_{0}^{4 / 3}= & -\frac{(4 / 3) \xi}{(2 / 3)(S t / \alpha) \chi+1} \frac{2(S t / 0.1)^{2 / 3} M_{\star}\left(3 M_{\star}\right)^{-2 / 3}}{k_{\mathrm{mig}} G c_{\mathrm{s} 1}^{-2} \mathrm{AU}^{-\zeta}} \\
& \times \frac{1}{1-\zeta}\left(r^{1-\zeta}-r_{0}^{1-\zeta}\right)
\end{aligned}
$$

Here $M_{0}$ and $r_{0}$ are the starting mass and starting location of the protoplanet, respectively. We can now divide the equation by its solution at $r=0, M(0)=M_{\max }$, to obtain

$$
\frac{M^{4 / 3}-M_{0}^{4 / 3}}{M_{\max }^{4 / 3}-M_{0}^{4 / 3}}=1-\left(\frac{r}{r_{0}}\right)^{1-\zeta} \text {. }
$$

The "maximum mass" reached at $r=0$ for $\zeta<1$ is given by

$$
\begin{aligned}
M_{\max }^{4 / 3}= & M_{0}^{4 / 3}+\frac{(4 / 3) \xi}{(2 / 3)(S t / \alpha) \chi+1} \frac{2(S t / 0.1)^{2 / 3} M_{\star}\left(3 M_{\star}\right)^{-2 / 3}}{k_{\mathrm{mig}} G c_{\mathrm{s} 1}^{-2} \mathrm{AU}^{-\zeta}} \\
& \times \frac{r_{0}^{1-\zeta}}{1-\zeta} .
\end{aligned}
$$

For $\zeta>1$, the aspect ratio $H / r$ increases when approaching the star (Eq. (6)) and migration is stalled by the high temperature in the inner regions of the protoplanetary disc. The protoplanet therefore never reaches $r=0$ in that case. We can reformulate Eq. (22) as a scaling law for $M_{\max }$ in the limit $M_{\max } \gg M_{0}$,

$$
\begin{aligned}
M_{\max }= & 11.7 M_{\mathrm{E}} \frac{(S t / 0.01)^{1 / 2}}{\{[(2 / 3)(S t / \alpha) \chi+1] / 2.9\}^{3 / 4}}\left(\frac{\xi}{0.01}\right)^{3 / 4} \\
& \times\left(\frac{M_{\star}}{M_{\odot}}\right)^{1 / 4}\left(\frac{k_{\mathrm{mig}}}{4.42}\right)^{-3 / 4}\left(\frac{c_{\mathrm{s} 1}}{6.5 \times 10^{2} \mathrm{~m} \mathrm{~s}^{-1}}\right)^{3 / 2} \\
& \times\left(\frac{1-\zeta}{4 / 7}\right)^{-1}\left(\frac{r_{0}}{25 \mathrm{AU}}\right)^{(3 / 4)(1-\zeta)} .
\end{aligned}
$$

We see here how $\xi$, which sets the pebble accretion rate, and $k_{\text {mig }}$, which sets the migration rate, pull the maximum mass in opposite directions at an exactly equal power index. Any reduction in the pebble accretion rate, for example from 3D accretion if the pebble scale height is larger than $10 \%$ of the gas scale height in Eq. (2), will act in similar way as a reduction in $\xi$ to reduce $M_{\max }$. We assumed in the derivations above that $k_{\text {mig }}$ is a constant. This assumption may break down in the inner, viscously heated regions of the protoplanetary disc (typically interior of $1 \mathrm{AU}$ for nominal accretion rates and disc masses) where the positive corotation torque can slow down or reverse migration (Bitsch \& Johansen 2016; Brasser et al. 2017). We note also that the weak scaling with $M_{\star}^{1 / 4}$ in Eq. (23) does not take into account that $c_{\mathrm{s} 1}$ depends on the luminosity, and hence on the mass, of the $s^{2}{ }^{2}$.

2 Assuming that the luminosity of the host star has a power-law dependence on the stellar mass, $L_{\star} \propto M_{\star}^{p}$, yields a temperature at $1 \mathrm{AU}$ that scales as $T_{1} \propto L_{\star}^{2 / 7} M_{\star}^{-1 / 7} \propto M_{\star}^{(2 p-1) / 7}$ (Ida et al. 2016) and hence $c_{\mathrm{s} 1} \propto M_{\star}^{(2 p-1) / 14}$. This gives a combined mass-dependence of Eq. (23) as $M_{\max } \propto M_{\star}^{1 / 4+(3 p-3 / 2) / 14}$, which is close to linear for $p=3$. 


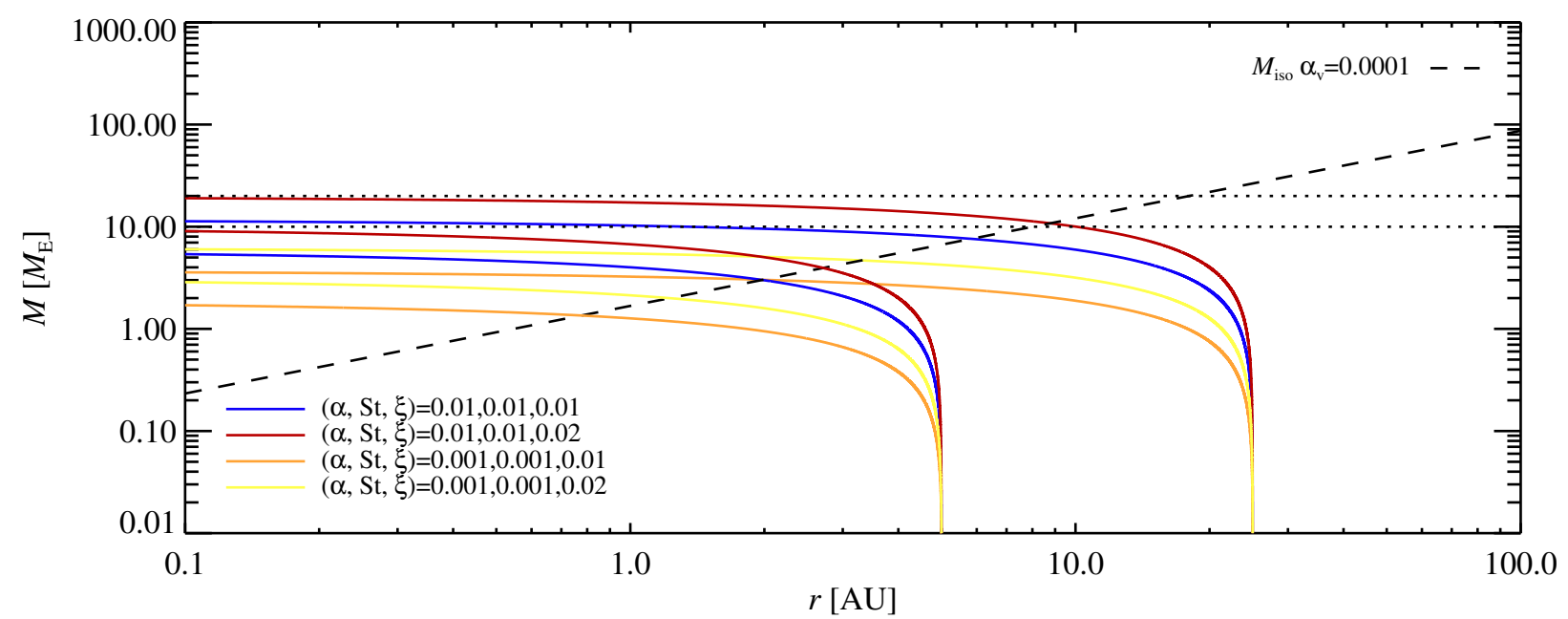

Fig. 1. Analytical growth tracks of planetary cores for four combinations of the accretion viscosity $\alpha$, the pebble Stokes number $S t$ (set to be equal to $\alpha$ ), and the ratio of radial pebble-to-gas flux rates $\xi$. Protoplanets are started at either 5 or $25 \mathrm{AU}$ and with a starting mass of $0.01 M_{\mathrm{E}}$. The pebble isolation mass is indicated for two values of the turbulent viscosity $\alpha_{\mathrm{v}}$, assumed here to be 0.1 times the accretion viscosity $\alpha$. The proportionality between the migration rate and the mass results in all growth tracks turning nearly horizontal before reaching their maximal mass at $r=0$.

We finally obtain the shape of the growth track, $r(M)$, from Eq. (21),

$r(M)=r_{0}\left(1-\frac{M^{4 / 3}-M_{0}^{4 / 3}}{M_{\max }^{4 / 3}-M_{0}^{4 / 3}}\right)^{1 /(1-\zeta)}$.

In Appendix B we use this expression to derive the time associated with each step in the growth track. We show analytical growth tracks in Fig. 1, for pairs of values of $\alpha=S t$ and $\xi$. We use a disc temperature profile here with $c_{\mathrm{s} 1}=650 \mathrm{~m} \mathrm{~s}^{-1}$ and $\zeta=3 / 7$, appropriate for the outer regions of the protoplanetary disc where viscous heating is negligible (Chiang \& Youdin 2010; Bitsch et al. 2015b; Ida et al. 2016). The growth tracks start off nearly vertical (growth dominates over migration), but as the migration rate increases, eventually the growth tracks turn horizontal and reach $M_{\max }$ after migrating to $r=0$. Type-I migration is truly a tough opponent for the planetary core to overcome.

\subsection{Pebble isolation mass}

The growth stage of the core ends as the planetary core reaches the pebble isolation mass. Here the gravity of the protoplanet perturbs the gas flow enough to form a plateau of Keplerian motion on the exterior side of the orbit, trapping the migrating pebbles there. The lack of heating by infalling pebbles then allows the gas to decrease its entropy by radiative heat loss and contract slowly to accrete a growing envelope around the core (Lambrechts et al. 2014). In Bitsch et al. (2018b) the pebble isolation mass was fitted to $3 \mathrm{D}$ simulations by the expression

$$
\begin{aligned}
M_{\text {iso }}= & 25 M_{\mathrm{E}}\left[\frac{H / r}{0.05}\right]^{3}\left[0.34\left(\frac{\log \left(\alpha_{3}\right)}{\log \left(\alpha_{\mathrm{v}}\right)}\right)^{4}+0.66\right] \\
& \times\left[1-\frac{\partial \ln P / \partial \ln r+2.5}{6}\right] .
\end{aligned}
$$

Here $\alpha_{3}=10^{-3}$ is a constant and $\alpha_{\mathrm{v}}$ is the turbulent viscosity, which we distinguish in this paper from the $\alpha$-value of the mass-accretion rate of Eq. (7), as the latter may be driven by disc winds at a weak level of actual turbulence (Bai \& Stone 2013; Béthune et al. 2017). Dürmann \& Kley (2015) showed that the gas flow through the protoplanetary disc achieves a constant rate through a planetary gap and that the migration rate is not dependent on the global gas flow. We therefore here assume that the global gas speed also does not affect gap formation and use a nominal value of $\alpha_{\mathrm{v}}=10^{-4}$ to calculate the pebble isolation mass in Eq. (25) (see Armitage et al. 2013; Hasegawa et al. 2017, for a discussion of this distinction). We motivate our usage of $\alpha_{\mathrm{v}}$ in defining the pebble isolation mass by noting that the gas speed driven by the turbulent viscosity over the length scale of the gap, assumed to be the gas scale height $H$, is

$u_{\mathrm{v}} \sim \frac{v}{H} \sim(H / r)^{-1} u_{r}$,

where $u_{r}$ is the global gas-accretion speed. Hence turbulent viscosity is expected to be a factor $(H / r)^{-1}$ times more effective than the global gas accretion speed at counteracting gap formation. However, we emphasize that Dürmann \& Kley (2015) did not distinguish between the $\alpha$ measuring disc accretion and the $\alpha_{\mathrm{v}}$ measuring turbulent viscosity. Future studies are needed to assess the effect of the global gas accretion speed, here parameterised through $\alpha$, on gap formation and pebble isolation mass.

The isolation mass for the nominal value of $\alpha_{\mathrm{v}}$ is overplotted in Fig. 1. The pebble isolation mass is increased over the expression given in Eq. (25) for small pebbles or strong particle turbulence (Bitsch et al. 2018b; Ataiee et al. 2018); we ignore such effects here since we work in the limit of weak turbulence and since even small pebbles of $S t=0.01$ are easily trapped at the outer edge of the gap.

\subsection{Reaching the pebble isolation mass}

One can derive analytically the location where the protoplanet growth track crosses the pebble isolation mass. The pebble isolation mass changes as $r$ decreases from its initial $r_{0}$. Scaling Eq. (25) from the starting position of the growth track, $r_{0}$, we obtain the expression

$M_{\mathrm{iso}}(r)=M_{\mathrm{iso}, 0}\left(\frac{r}{r_{0}}\right)^{(3 / 2)(1-\zeta)}$. 
Here $M_{\mathrm{iso}, 0}$ is the isolation mass at $r=r_{0}$. In the limit $M \gg M_{0}$ the protoplanet therefore reaches pebble isolation mass at $r=r_{\text {iso }}$ when

$M_{\text {iso }}^{4 / 3}=M_{\text {iso }, 0}^{4 / 3}\left(\frac{r_{\text {iso }}}{r_{0}}\right)^{2(1-\zeta)}=M_{\max }^{4 / 3}\left[1-\left(\frac{r_{\text {iso }}}{r_{0}}\right)^{1-\zeta}\right]$.

This equation forms a second-order polynomial,

$\left(\frac{M_{\mathrm{iso}, 0}}{M_{\max }}\right)^{4 / 3} X^{2}+X-1=0$,

with $X=\left(r_{\text {iso }} / r_{0}\right)^{1-\zeta}$ or $\Delta r_{\text {iso }}=r_{0}-r_{\text {iso }}=\left[1-X^{1 /(1-\zeta)}\right] r_{0}$. The positive solution to the polynomial is

$X=\frac{\sqrt{1+4 A}-1}{2 A}$,

where $A=\left(M_{\text {iso }, 0} / M_{\text {max }}\right)^{4 / 3}$. We note that a solution exists for all values of $A$, that is the pebble isolation mass is reached for all starting positions $r_{0}$. This is due to the steeply falling pebble isolation mass with decreasing distance, in the passively irradiated case. Our results generally do not apply to the viscously heated interior regions of the protoplanetary disc where $\zeta$ increases and the aspect ratio becomes relatively constant (Bitsch et al. 2015b; Ida et al. 2016; Brasser et al. 2017).

In the limit $A \gg 1$, corresponding to $r_{0} \gg r_{\text {iso }}$ through Eq. (28), Eq. (30) has the limiting solution

$X=A^{-1 / 2}$,

and hence the isolation mass is reached at radius

$r_{\text {iso }} / r_{0}=A^{-(1 / 2)[1 /(1-\zeta)]}$.

We can furthermore make use of $M_{\mathrm{iso}, 0} \propto r_{0}^{(3 / 2)(1-\zeta)}$ and $M_{\max } \propto$ $r_{0}^{(3 / 4)(1-\zeta)}$ to infer $A=\left(M_{\text {iso, } 0} / M_{\max }\right)^{4 / 3} \propto r_{0}^{1-\zeta}$. That gives now the simple relation

$r_{\text {iso }} \propto r_{0}^{1 / 2}$.

The length over which the protoplanet migrates before reaching pebble isolation mass is therefore a steeply increasing function of the starting position. In the same limit $\left(r_{0} \gg r_{\text {iso }}\right)$ the reached pebble isolation mass becomes simply $M_{\text {iso }}=M_{\max }$, by inserting $r_{\text {iso }} / r_{0}$ in Eq. (28). This simple result arises because the growth track turns nearly horizontal after a significant migration distance and hence the core is close to its maximum mass $M_{\max }$ when it finally reaches pebble isolation mass.

In Fig. 2 we plot the calculated distance at which a protoplanet reaches isolation mass, $r_{\text {iso }}$, as a function of the starting position, $r_{0}$, for different values of $\alpha=S t$ and $\xi$. The distance is obtained from the full solution of Eq. (29). For a core to reach isolation mass in the 5-10 AU region, the protoplanet must generally start beyond $20 \mathrm{AU}$, for nominal values of $\xi=0.01-0.02$. Nearly in situ core assembly by pebble accretion requires much higher values of $\xi \gtrsim 0.05-0.1$.

\section{Growth tracks including gas accretion}

We now include the effect of gap formation and gas accretion on planetary growth tracks. We turn to numerical integration, since the reduction of the migration rate and gas-accretion rate by gap formation render the governing equations much more complex than in the previous section.

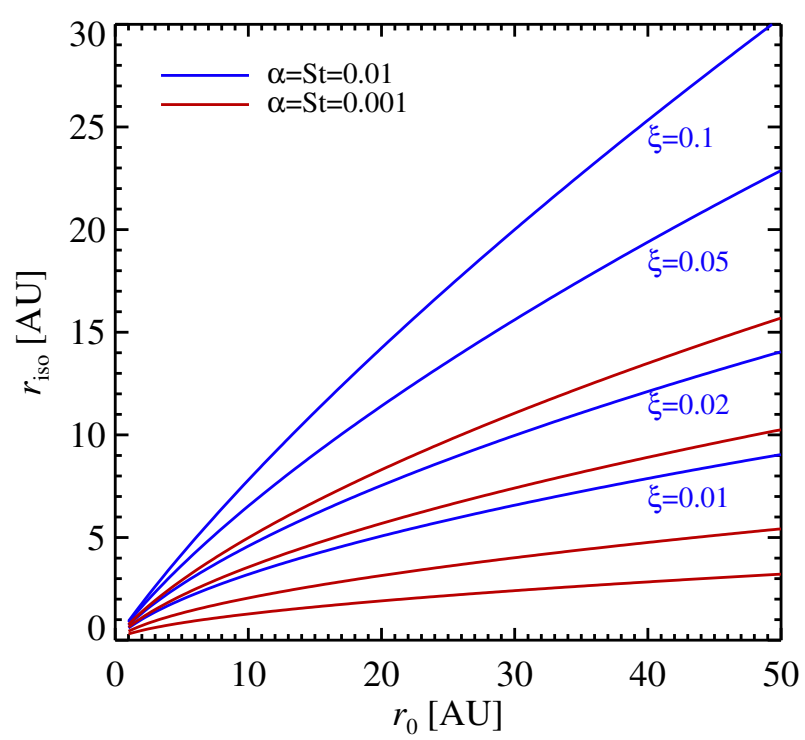

Fig. 2. Radial location of reaching the pebble isolation mass, $r_{\text {iso }}$, as a function of the starting position in the disc, $r_{0}$, for different values of the pebble-to-gas flux ratio $\xi$. For low values of $\xi$, reaching the pebble isolation mass in the 5-10 AU region requires migration over tens of AU. Nearly in situ assembly of a planetary core is possible when the pebble-to-gas flux ratio $\xi \gtrsim 0.05-0.1$.

\subsection{Type-I migration and relative gap height}

Kanagawa et al. (2018) performed a suite of 2D simulations to measure the torque on embedded planets of a wide range of masses. They found that the torque is well described by the classical type-I torque, which gives rise to the migration rate expression given in Eq. (3), multiplied by the relative gap height,

$\Gamma=-c_{\mathrm{mig}} \Gamma_{0} \frac{\Sigma_{\mathrm{gap}}}{\Sigma_{\mathrm{g}}}$.

Here $c_{\text {mig }}=k_{\text {mig }} / 2$ is the torque prefactor, $\Sigma_{\text {gap }}$ is the surface density in the gap, $\Sigma_{\mathrm{g}}$ is the unperturbed surface density, and $\Gamma_{0}\left(\propto M^{2}\right)$ is the natural torque scaling. The surface density at the bottom of the gap is fitted well by the expression (Duffell \& MacFadyen 2013; Fung et al. 2014; Kanagawa et al. 2015; Fung \& Chiang 2016)

$\frac{\Sigma_{\text {gap }}}{\Sigma_{\mathrm{g}}}=\frac{1}{1+0.04 K}$,

where

$K=\left(\frac{M}{M_{\star}}\right)^{2}\left(\frac{H}{r}\right)^{-5} \alpha_{\mathrm{v}}^{-1}$.

This implies that the migration rate, $\dot{r}=2 \Gamma /\left(M v_{\mathrm{K}}\right)$, falls as $1 / M$ above the gap transition mass $M_{\text {gap }}$ (defined as the mass for which $K=1 / 0.04$ ). The gap transition mass and the pebble isolation mass are actually closely related concepts. While the gap transition mass measures the mass required to make a relative gap height of 0.5 , the pebble isolation mass measures the first appearance of a point of zero pressure gradient at the outside of the planetary gap. The latter criterion is slightly easier to fulfill, as a relative gap height of around 0.85 is sufficient to invert the pressure gradient in the simulations of Bitsch et al. (2018b, we inferred this relative gap height from their Fig. A.1). 

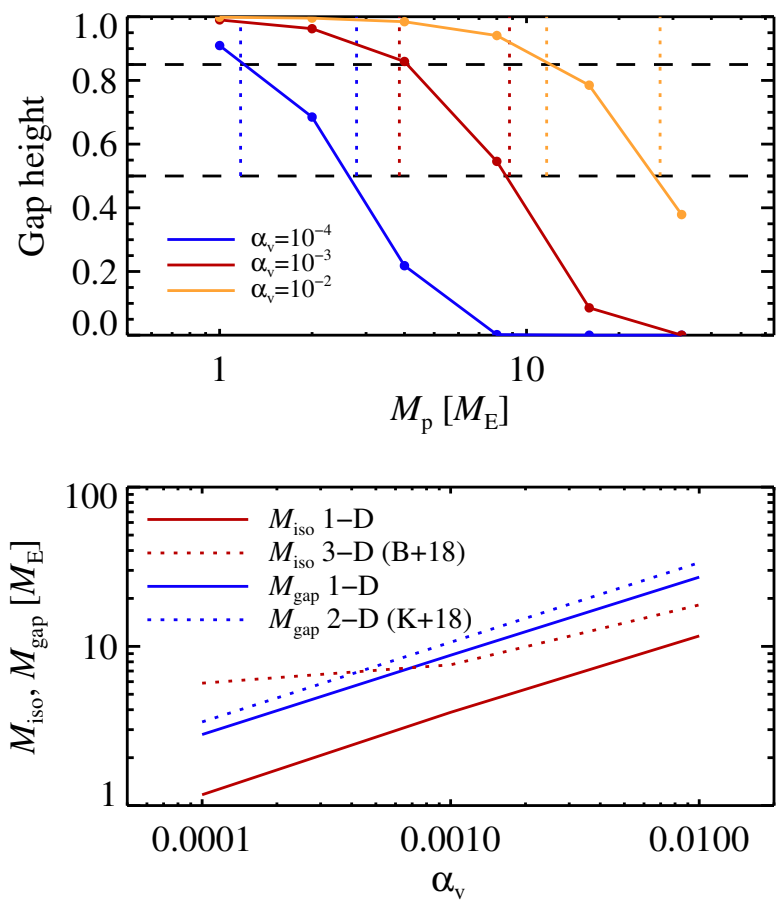

Fig. 3. Results of 1D simulations of an accretion disc with an embedded planet whose torque on the gas is based on the parametrisations of D'Angelo \& Lubow (2010). Top panel: equilibrium relative gap height as a function of the planetary mass for three values of the viscous $\alpha_{\mathrm{v}}$. The dashed lines mark the $50 \%$ relative gap height and the $85 \%$ relative gap height, the latter approximately corresponding to the pebble isolation mass. The dotted lines show the measured pebble isolation mass and $50 \%$ gap mass. Bottom panel: pebble isolation mass $\left(M_{\text {iso }}\right)$ and the gap transition mass $\left(M_{\text {gap }}\right)$ as a function of $\alpha_{\mathrm{v}}$. We find generally that $M_{\text {gap }} \approx 2.3 M_{\text {iso. }}$. The measured gap transition mass corresponds well to the 2D simulations of Kanagawa et al. (2018), while the measured pebble isolation mass is about a factor two lower than reported in Bitsch et al. (2018b) and displays a more consistent drop with lower $\alpha_{\mathrm{v}}$.

To check the robustness of the relative gap height needed for pebble isolation, we performed additional 1D simulations of an accretion disc with an embedded planet, varying the turbulent viscosity. The torque from the planet was mimicked using the torque profile of D'Angelo \& Lubow (2010), based on their 3D simulations. The simulations were run until the relative gap height reached equilibrium between the gap-opening torque and the viscous momentum transport $\left(10^{3}, 10^{4}, 10^{5} \mathrm{yr}\right.$ for $\alpha_{\mathrm{v}}=10^{-2}$, $10^{-3}, 10^{-4}$, respectively). The results are shown in Fig. 3 and confirm that the pebble isolation mass is reached at a relative gap height of 0.85 . We find a general scaling that the gap transition mass, that is the mass of $50 \%$ relative gap height, is 2.3 times the pebble isolation mass. The measured gap transition mass agrees well with the relative gap height scaling in Eqs. (35) and (36), and, in particular, that the gap transition mass scales with the square root of $\alpha_{\mathrm{v}}$.

We nevertheless encountered some discrepancy between the $\alpha_{\mathrm{v}}$-dependence of the pebble isolation mass inferred from our simulations and those of Bitsch et al. (2018b). At high and medium $\alpha_{\mathrm{v}}\left(10^{-2}\right.$ and $\left.10^{-3}\right)$ our 1D pebble isolation mass is approximately a factor two lower than the $3 \mathrm{D}$ pebble isolation mass of Bitsch et al. (2018b). It is nevertheless well-known that gaps formed by a 1D torque prescription become artificially deeper than what is found in 2D and 3D simulations (Hallam \& Paardekooper 2017). For low $\alpha_{\mathrm{v}}\left(10^{-4}\right)$ the pebble isolation mass of Bitsch et al. (2018b) furthermore showed a weak logarithmic scaling with $\alpha_{\mathrm{v}}$ (Eq. (25)), while our 1D pebble isolation mass maintains its proportionality to the square root of $\alpha_{\mathrm{v}}$, similar to the scaling of the gap transition mass with $\alpha_{\mathrm{v}}$ (Eqs. (35) and (36)). This difference could be due to (1) Rossby wave instabilities triggered at the gap edge at low $\alpha_{\mathrm{v}}$ in 3D simulations or (2) to the fact that the simulations of Bitsch et al. (2018b) were run for only 1000 orbital timescales at the planet position. The 2D simulations of Ataiee et al. (2018) also show a somewhat weaker dependence of the pebble isolation mass on $\alpha_{\mathrm{v}}$ than the square root dependence that we find in Fig. 3. Identifying the actual reason for the discrepancy will require a dedicated study beyond the scope of the current paper. In our nominal model we therefore set the pebble isolation mass according to Bitsch et al. (2018b) and the gap transition mass to 2.3 times the pebble isolation mass. This yields a modified migration equation

$\dot{r}=\frac{\dot{r}_{\mathrm{I}}}{1+\left[M /\left(2.3 M_{\mathrm{iso}}\right)\right]^{2}}$,

where $\dot{r}_{\mathrm{I}}$ is the classical type-I migration rate and $M_{\text {iso }}$ is the pebble isolation mass given in Eq. (25). We also show results in Sect. 3.8 where we use (a) $M_{\text {gap }}$ from the $2 \mathrm{D}$ simulations of Kanagawa et al. (2018) and $M_{\text {iso }}$ from the 3D simulations of Bitsch et al. (2018b) and (b) $M_{\text {gap }}$ from the 2D simulations and $M_{\text {iso }}=M_{\text {gap }} / 2.3$.

Reaching first the pebble isolation mass and, shortly after, the gap transition mass thus signifies three important mile-stones for the growth of a protoplanet: (a) the end of the accretion of pebbles, (b) the beginning of gas contraction, and (c) the transition to a slow-down in the migration caused by the deepening gap.

\subsection{Gas accretion}

The gas-accretion rate of a protoplanet embedded in a protoplanetary disc is highly uncertain. Even the term accretion is, in our opinion, slightly misleading, as the mass increase takes place in the form of a contraction of the envelope due to entropy reduction by radiative heat loss. Klahr \& Kley (2006) demonstrated that the circumplanetary disc formed in some isothermal simulations is replaced by a hot, hydrostatic gas blob in simulations including compressive heating and radiative transfer. Ikoma et al. (2000) performed 1D simulations of the envelope contraction and found runaway contraction where the energy loss, and hence contraction rate, is accelerated at higher envelope masses. D'Angelo \& Bodenheimer (2013) presented extensive simulations of the gas contraction onto low-mass cores and found, as in earlier 1D work, that the contraction is accelerated at higher core mass (the same trend was observed in Lambrechts \& Lega 2017). Lambrechts et al. (2018) measured the gas-contraction rate in hydrodynamical simulations with radiative transfer for planetary cores of up to Jupiter mass. They found that the accretion rate is orders of magnitude lower than the mass flux passing through the Hill radius, as most of this gas is transported out again on horseshoe orbits and more complex streamlines.

We use here the gas-accretion prescription proposed in Ida et al. (2018), taking into account both the Kelvin-Helmholtzlike contraction of the envelope and the feeding of gas from the protoplanetary disc into the Hill sphere of the protoplanet. The contraction of the gaseous envelope is assumed to commence after the core reaches pebble isolation mass, at a rate motivated by Ikoma et al. (2000),

$\left(\frac{\mathrm{d} M}{\mathrm{~d} t}\right)_{\mathrm{KH}}=10^{-5} M_{\mathrm{E}} \mathrm{yr}^{-1}\left(\frac{M}{10 M_{\mathrm{E}}}\right)^{4}\left(\frac{\kappa}{0.1 \mathrm{~m}^{2} \mathrm{~kg}^{-1}}\right)^{-1}$. 
Here $\kappa$ is the opacity of the envelope, which we discuss further below. This accretion rate will become higher than what the protoplanetary disc can supply for high planetary masses. Tanigawa \& Tanaka (2016) used isothermal, global simulations to demonstrate that gas enters the Hill sphere at a rate

$$
\begin{aligned}
\left(\frac{\mathrm{d} M}{\mathrm{~d} t}\right)_{\mathrm{disc}}= & \frac{0.29}{3 \pi}\left(\frac{H}{r}\right)^{-4}\left(\frac{M}{M_{\star}}\right)^{4 / 3} \frac{\dot{M}_{\mathrm{g}}}{\alpha} \frac{\Sigma_{\text {gap }}}{\Sigma_{\mathrm{g}}} \\
= & 1.5 \times 10^{-3} M_{\mathrm{E}} \mathrm{yr}^{-1}\left(\frac{H / r}{0.05}\right)^{-4}\left(\frac{M}{10 M_{\mathrm{E}}}\right)^{4 / 3} \\
& \times\left(\frac{\alpha}{0.01}\right)^{-1}\left(\frac{\dot{M}_{\mathrm{g}}}{10^{-8} M_{\odot} \mathrm{yr}^{-1}}\right) \frac{1}{1+\left(M / M_{\text {gap }}\right)^{2}} .
\end{aligned}
$$

This equation is derived in the companion paper by Ida et al. (2018) - we note here that the simpler form, $(\mathrm{d} M / \mathrm{d} t)_{\mathrm{disc}} \propto$ $R_{\mathrm{H}}^{2} \Omega \Sigma_{\text {gap }}\left(R_{\mathrm{H}} / H\right)^{2}$, shows that the equation can be decomposed into the full flux into the Hill sphere multiplied by the squared ratio of the Hill radius to the scale-height. The latter reflects an empirical finding that the protoplanet increases its ability to accrete the gas streamlines that enter the Hill radius as the mass increases (Tanigawa \& Tanaka 2016).

The prefactor in Eq. (39) can be converted to solar units to compare with the disc accretion rate, $1.5 \times 10^{-3} M_{\mathrm{E}} \mathrm{yr}^{-1} \approx 4.5 \times$ $10^{-9} M_{\odot} \mathrm{yr}^{-1}$. If the planet contracts rapidly enough to absorb the entire gas flow into the Hill radius, then we must therefore limit the accretion rate to the global gas-accretion rate (see Lubow \& D'Angelo 2006, for a discussion of this limit). We therefore set the gas-accretion rate of the planet equal to

$$
\left(\frac{\mathrm{d} M}{\mathrm{~d} t}\right)_{\mathrm{g}}=\min \left[\left(\frac{\mathrm{d} M}{\mathrm{~d} t}\right)_{\mathrm{KH}},\left(\frac{\mathrm{d} M}{\mathrm{~d} t}\right)_{\mathrm{disc}}, \dot{M}_{\mathrm{g}}\right] \text {. }
$$

Ikoma et al. (2000) found that the Kelvin-Helmholtz gasaccretion rate increases inversely proportionally to the opacity $\kappa$ (Eq. 38). Bitsch \& Johansen (2016) calculated an opacity of micron-sized ice particles in the range $0.01-0.1 \mathrm{~m}^{2} \mathrm{~kg}^{-1}$. For core accretion by pebble accretion, we expect that the accreted gas will be completely pebble-free beyond the pebble isolation mass (Lambrechts et al. 2014). Small dust can nevertheless pass the planet barrier together with the accreted gas, constituting maybe $10 \%$ of the total content of solids. That gives a nominal opacity in the range $0.001-0.01 \mathrm{~m}^{2} \mathrm{~kg}^{-1}$. The opacity could be even lower if the grains in the envelope coagulate and sediment (Mordasini 2014). We adopt here a standard opacity value of $\kappa=0.005 \mathrm{~m}^{2} \mathrm{~kg}^{-1}$.

\subsection{Protoplanetary disc model}

The growth rates reported in Ikoma et al. (2000) were measured at a single value of the gas surface density and gas temperature at the outer boundary. Hence the dependence of the accretion rates on the gas surface density and temperature is not known. If the gas contraction is indeed limited by the ability of the envelope to cool, then the outer boundary condition may be of little significance (as found by Piso \& Youdin 2014; Lee \& Chiang 2015). This in turn implies that mass accretion wins more easily over migration when the gas disc is depleted compared to the primordial value, since the initial gas-accretion rate is relatively unaffected by the protoplanetary disc surface density, while the migration rate is proportional to the surface density ${ }^{3}$.

3 The reduced migration rate at low gas column densities is an important component of the model of super-Earth formation in depleted gas discs presented in Lee \& Chiang (2016).
The dependence of the migration rate on the gas surface density, combined with the non-dependence of the KelvinHelmholtz contraction rate on the surface density, therefore necessitates a specific disc evolution model to integrate the growth tracks. We adopt here a nominal viscous accretion disc model where $\dot{M}_{\mathrm{g}}$ evolves from $10^{-7}$ to $10^{-8} M_{\odot} \mathrm{yr}^{-1}$ over $3 \mathrm{Myr}$ (typical for solar-mass stars, see Hartmann et al. 2016). Following Hartmann et al. (1998), the accretion rate onto the star evolves as

$\dot{M}_{\mathrm{g}}(t)=\dot{M}_{0}\left[\frac{t}{t_{\mathrm{s}}}+1\right]^{-(5 / 2-\gamma) /(2-\gamma)}$,

where $\gamma$ is the power-law index of the turbulent viscosity, $v \propto r^{\gamma}$, and the characteristic timescale, $t_{\mathrm{s}}$, is

$t_{\mathrm{s}}=\frac{1}{3(2-\gamma)^{2}} \frac{R_{1}^{2}}{v_{1}}$.

Here $R_{1}$ is the initial characteristic disc size and $v_{1}$ is the value of the viscosity at that radius. Knowing the temperature power-law index $\zeta$ results in $\gamma=3 / 2-\zeta(=15 / 14$ for $\zeta=3 / 7)$. We proceed by choosing $R_{1}$ to yield the desired starting and ending mass-accretion rate over an assumed disc lifetime of $3 \mathrm{Myr}$. In Sect. 3.7, we present planet formation results for protoplanetary discs with initially smaller sizes and hence a steeper temporal decay of their mass-accretion rates.

We take into account in the numerical simulations that the pebble-accretion rate is lowered when the pebble scale height is higher than the pebble-accretion radius, following the method described in Johansen et al. (2015). Johansen \& Klahr (2005) demonstrated using MHD simulations that the turbulent viscosity $\alpha_{\mathrm{v}}$ and the turbulent diffusion coefficient $\delta$ are approximately equal; for simplicity we therefore assume $\delta=\alpha_{\mathrm{v}}$ in our simulations.

\subsection{Growth tracks with gas accretion}

The integration of the growth track bundles from Fig. 1 including gas accretion are shown in Fig. 4. We start the growth tracks at $t_{0}=0.9 \mathrm{Myr}$ and integrate until the disc is assumed to dissipate after $t=3 \mathrm{Myr}$. To demonstrate the effect of the starting time, we start one growth track (with $\alpha=S t=0.01$ and $\xi=0.01$ starting at $r_{0}=25 \mathrm{AU}$ ) at $t_{0}=0.3 \mathrm{Myr}$. The core growth tracks before reaching pebble isolation mass are relatively unaffected by the slow-down of the migration rate towards the gap transition mass (and follow the analytical solution that we derived in Sect. 2, even when we include here that the initial pebble accretion is reduced in 3D). However, after reaching the pebble isolation mass, the two growth tracks starting at 25 AU with $\alpha=S t=0.01$ turn quickly upwards, as the migration rate falls inversely proportional to the planetary mass. This results in gas accretion length scales that are much shorter than the core accretion length scale. In contrast to this, the growth tracks starting at $5 \mathrm{AU}$ reach an isolation mass of $M_{\text {iso }} \sim 1-5 M_{\mathrm{E}}$. At those core masses, Kelvin-Helmholtz contraction is very slow, according to Eq. (38), and these planets migrate towards the star with little success at accreting gas.

In Fig. 5 we show the final positions of protoplanets starting at 1-50 AU distance from the star in the protoplanetary disc and starting times between 0 and $3 \mathrm{Myr}$, for four values of the pebble-to-gas flux ratio $\xi$. The slow-down of migration in the gas-accretion phase allows more space for migration during the core-accretion stage, hence we can form gas giants at a lower pebble-accretion rate than in Bitsch et al. (2015a). For 


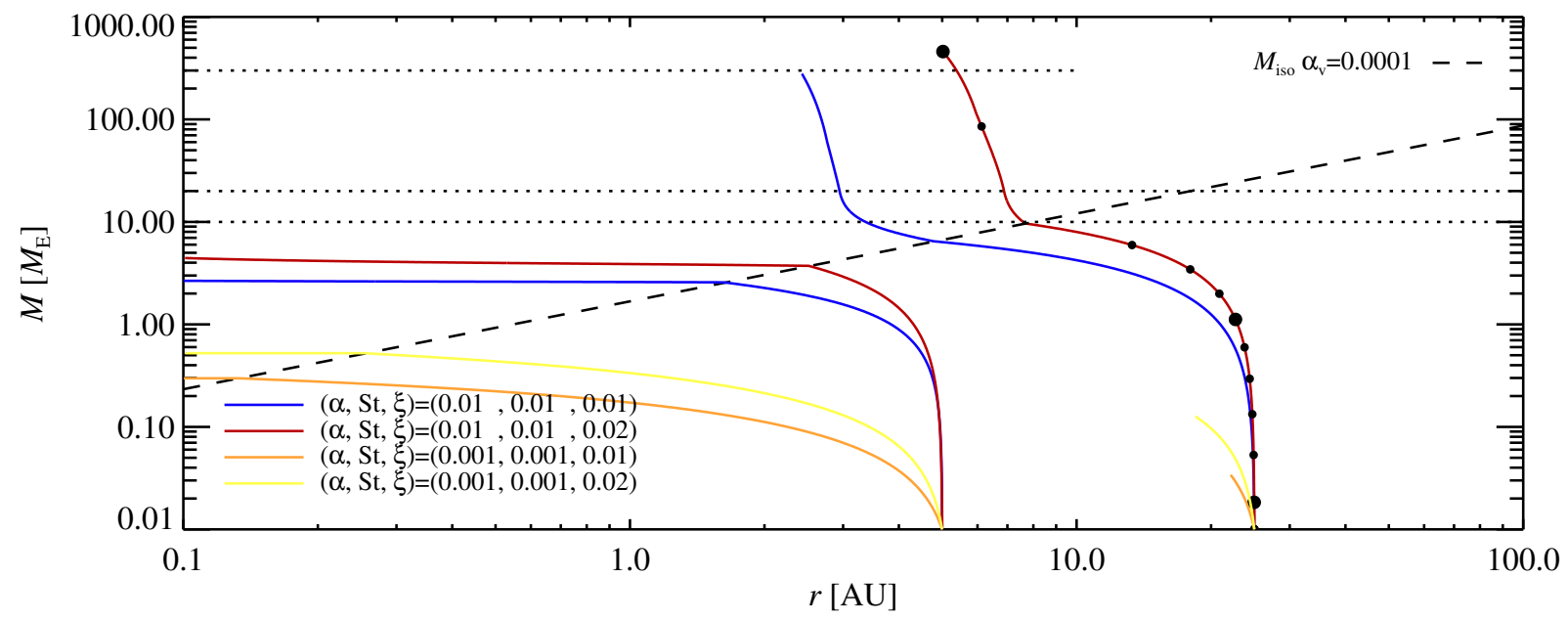

Fig. 4. Numerically integrated growth tracks for core accretion to the pebble isolation mass followed by gas accretion. We start the protoplanets at a mass of $M_{0}=0.01 M_{\mathrm{E}}$ at $t_{0}=0.9 \mathrm{Myr}$ (except for the $\alpha=S t=0.01$ growth track starting from $25 \mathrm{AU}$, which we start at $t_{0}=0.3 \mathrm{Myr}$ ) in a protoplanetary disc that evolves over a total time of 3 Myr. The dots indicate the time for the growth track that successfully forms a Jupiter analogue (small dots are separated by $0.2 \mathrm{Myr}$, large dots indicate a time of 3,2 and $1 \mathrm{Myr}$ ). We use a migration model where the migration rate is reduced by multiplication with the relative gap height, following Kanagawa et al. (2018). The migration rate is therefore inversely proportional to the planet mass beyond the pebble isolation mass. This results in gas accretion over a few astronomical units of migration.

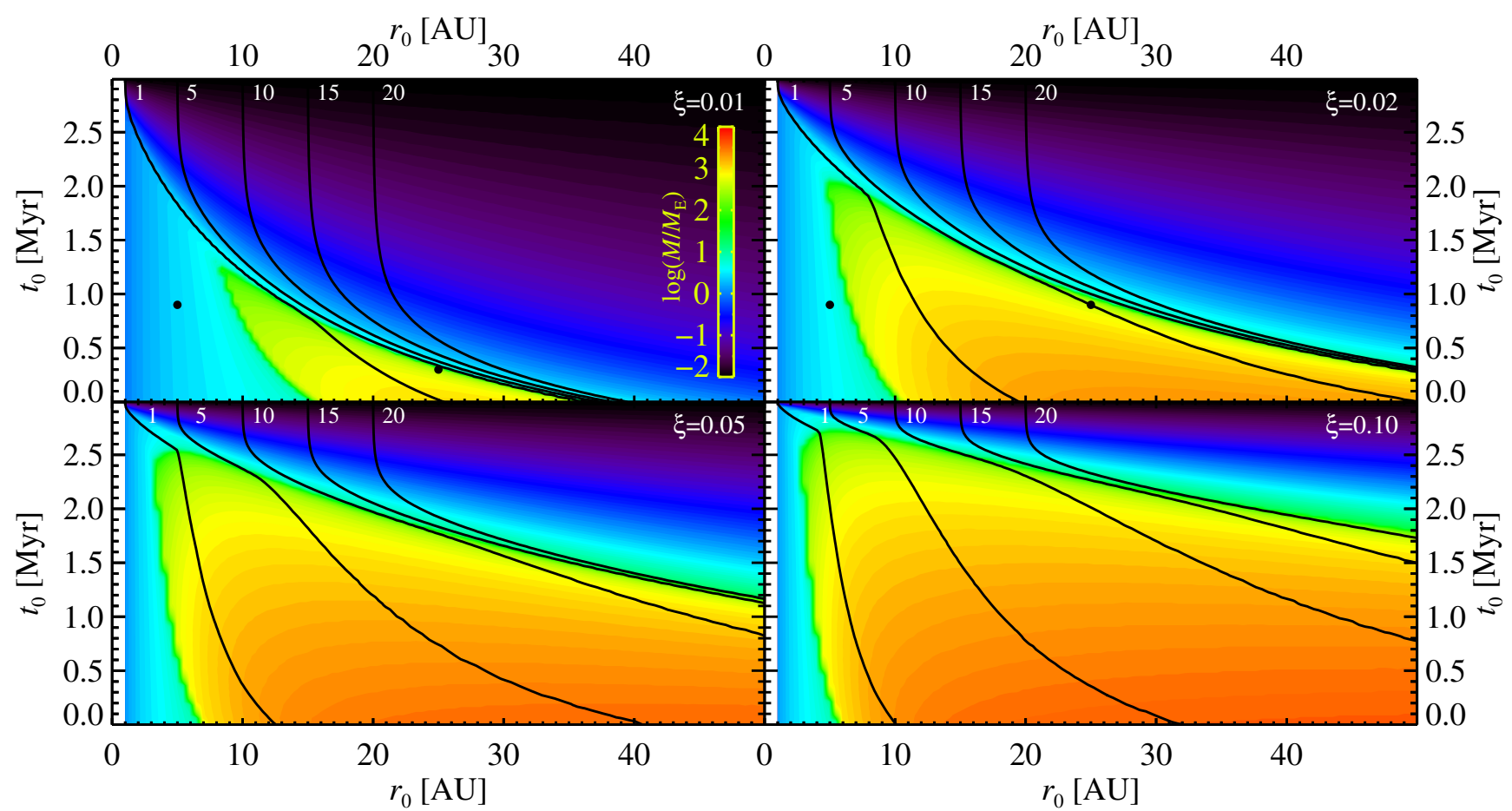

Fig. 5. Growth maps showing final masses (colours) and selected final positions (black contours) of protoplanets starting at 1-50 AU distance from the star between 0 and $3 \mathrm{Myr}$ of disc evolution. The four panels show the results for different values of the pebble-to-gas flux ratio $\xi$. The starting points of the selected growth tracks from Fig. 4 are indicated with dots. The cores of cold gas giants akin to Jupiter and Saturn start their assembly here in the 20-30 AU region for nominal values of $\xi=0.01-0.02$. Higher values of $\xi$ allow core assembly closer to the central star, starting in the 10-15 AU region.

nominal pebble-to-gas flux ratios of $\xi=0.01-0.02$, the cores of cold gas giants with final orbits in the 5-10 AU region (analogues of Jupiter and Saturn in the solar system) start their assembly much further out, in the 20-30 AU region. Shorter migration distances with starting locations in the 10-15 AU region are possible at higher pebble-to-gas flux ratios of $\xi=0.05-0.1$.

We find generally that our integrations produce much fewer hot and warm gas giants, with final orbits interior to $1 \mathrm{AU}$, compared to simulations that considered the traditional type-II migration (Bitsch \& Johansen 2016, 2017). This is due to the slow-down of the migration rate with the formation of a deep gap when using the Kanagawa et al. (2018) migration prescription here. Other studies have invoked photoevaporation as an effect to prevent massive planets from migrating all the way to the inner edge of the protoplanetary disc (Alexander \& Pascucci 2012; Ercolano \& Rosotti 2015); here and in Ida et al. (2018) we show that massive planets may be naturally prevented from 


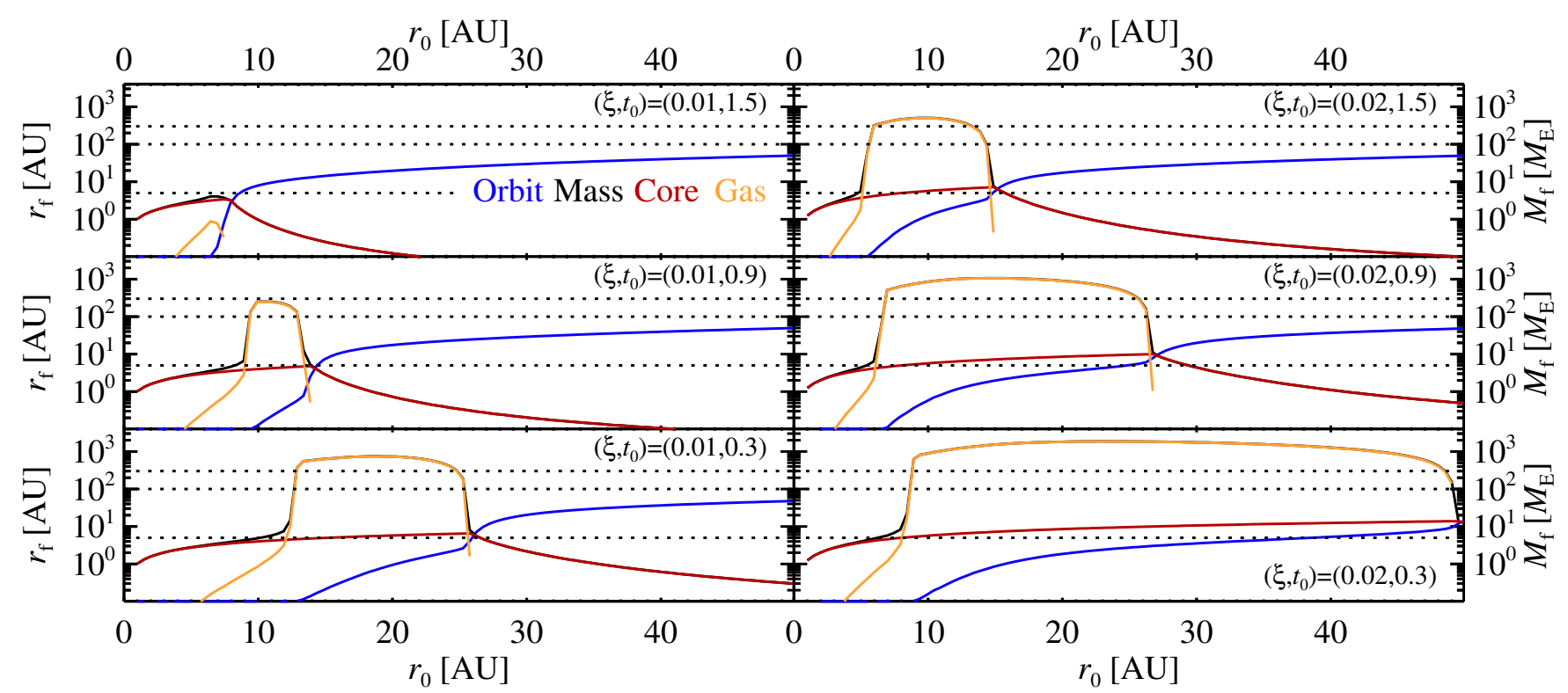

Fig. 6. Final orbits (left axis) and final total mass, core mass, and gas (right axis) for protoplanets starting from 1 to 50 AU (bottom/top axis), at three starting different times, $t_{0}=0.3,0.9,1.5 \mathrm{Myr}$ (bottom to top panels) and for two values of $\xi=0.01,0.02$ (left and right panels). The dotted lines indicate masses or orbits of 5, 100, and 300. Protoplanets starting far from the star experience both little growth and migration. As the starting position approaches the star, the core mass grows towards $10 M_{\mathrm{E}}$, triggering rapid migration and gas accretion. However, protoplanets that start inside $5 \mathrm{AU}$ have very protracted gas accretion due to the low pebble isolation mass there. The best analogues of Jupiter, Saturn, Uranus, and Neptune start their assembly in the region around $25 \mathrm{AU}$ for $\xi=0.02$ and $t_{0}=0.9 \mathrm{Myr}$.

migrating to the disc edge due to the deep gas gaps that they carve.

The core masses of our synthetic planets are nevertheless smaller than what is inferred for total content of heavy elements in the gas giants in the solar system. The recent data on the gravitational field of Jupiter by the Juno satellite yield a total amount of heavy elements between $25 M_{\mathrm{E}}$ and $45 M_{\mathrm{E}}$ (Wahl et al. 2017). However, we did not include in our simulations the contribution from planetesimal pollution during the gas-accretion phase. Shiraishi \& Ida (2008) modelled the pollution by late planetesimal infall numerically and found accretion of up to $10 M_{\mathrm{E}}$ of additional planetesimals, bringing the total amount of heavy elements more in line with observations.

The final orbits and masses for three selected starting times are shown in Fig. 6. One sees how the outcome of planet formation can be broadly divided into three categories. Protoplanets starting their growth far from the star experience both little growth and little migration and end as ice planets with masses broadly between 0.1 and $2 M_{\mathrm{E}}$ (as also found by Bitsch et al. 2015b). As the starting position is reduced, the cores grow towards the pebble isolation mass of $\approx 10 M_{\mathrm{E}}$. This triggers rapid gas accretion and the formation of Jupiter and Saturn analogues. The total gas mass plateaus at around $1000 M_{\mathrm{E}}$; this is an effect of gap formation that limits gas accretion in our gas accretion model that follows Tanigawa \& Tanaka (2016). Protoplanets that start less than approximately $5 \mathrm{AU}$ from the host star have low pebble isolation masses and therefore never experience gas accretion. These form analogues of the super-Earths found around many stars (Batalha et al. 2013), although we emphasize here that the mutual dynamics of trains of such migrating super-Earths, not included here, is key to understanding the final orbits and multiplicities of super-Earth-mass migrators (Izidoro et al. 2017; Matsumura et al. 2017). Alternatively, the orbital properties of observed super-Earths has been proposed to be consistent with a lack of planetary migration (Lee \& Chiang 2017). We also ignore here the truncation of the inner disc by the magnetospheric cavity, which may play an important role in reducing or halting migration (Liu et al. 2017). Our model does not readily explain how to stop the accretion of gas at the mass of Saturn or Jupiter, unless we invoke special timing of the dissipation of the gaseous protoplanetary disc. A possible solution to this problem is that the turbulent viscosity, $\alpha_{\mathrm{v}}$, is even lower than what we have assumed and that both the pebble isolation mass and gap transition mass are lower in real protoplanetary discs. An earlier onset of gap formation would be an efficient way to limit the flow of gas to the protoplanet, through Eq. (39).

\subsection{Formation of ice giants}

Forming planets with core masses equivalent to those of Uranus and Neptune in orbits of 10-20 AU (as is broadly consistent with the initial condition for the later planetesimal-driven migration of the giant planets; Gomes et al. 2005) is possible when a protoplanet starts slightly exterior to those that end up forming gas giants. However, the parameter space for forming ice giants is relatively small, since gas accretion is rapid for those core masses. If, on the other hand, the ice giants formed by giant impacts of a higher number of five-Earth-mass cores, as explored in Izidoro et al. (2015), then the seeds of the ice giants would be allowed to form even further from the Sun and not be in such a high risk of accreting substantial amounts of gas. Also, in our work we do not consider the release of water vapour in the gas envelope. The contraction of an envelope highly enriched in water vapour could explain the relatively small amount of hydrogen in the ice giants of the solar system (Lambrechts et al. 2014; Venturini \& Helled 2017).

\subsection{Population synthesis}

In Fig. 7 we illustrate the planetary populations arising from our models by performing a population-synthesis operation on the growth maps for $\xi=0.01$ and $\xi=0.02$. We sampled 10000 

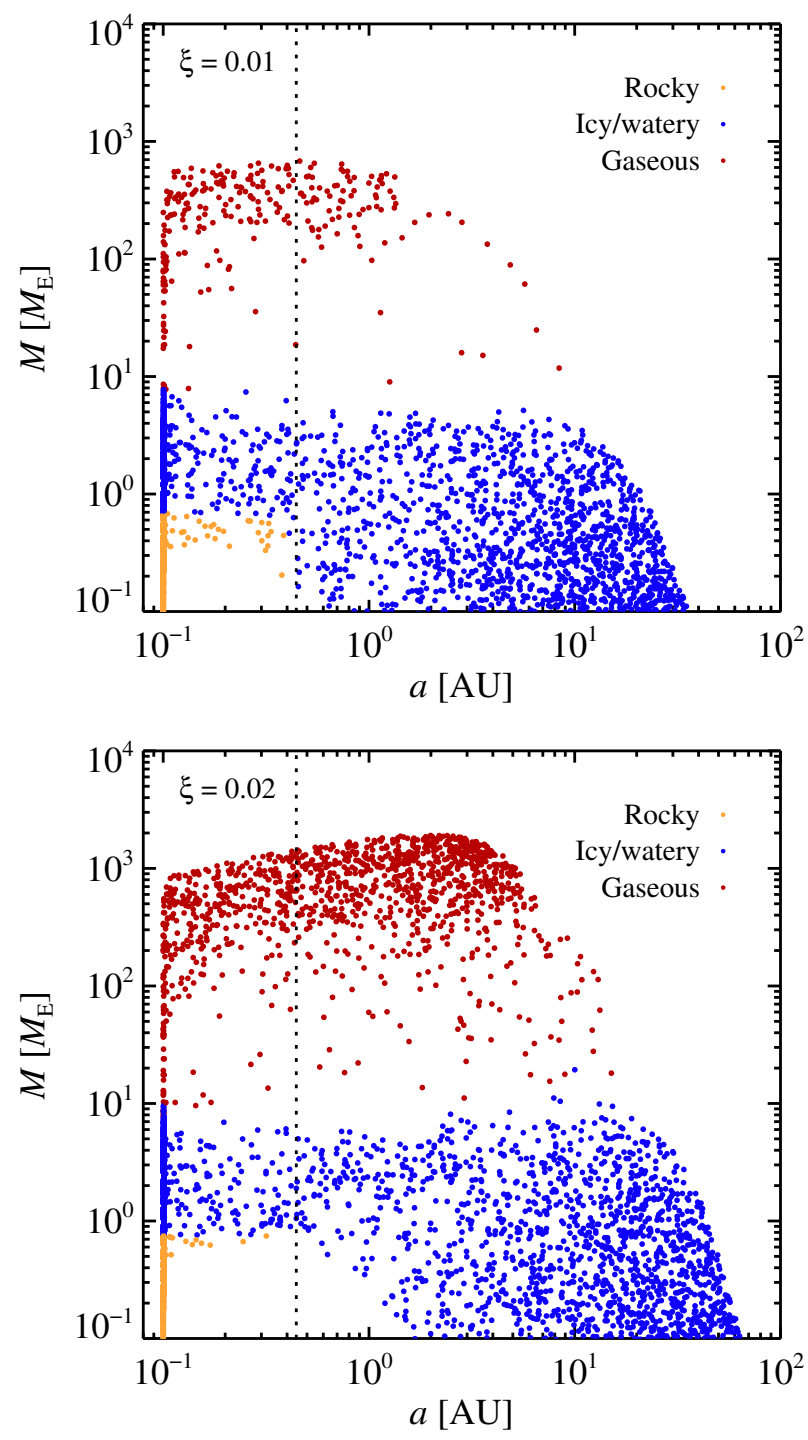

Fig. 7. Population synthesis of two of the growth maps shown in Fig. 5. We have selected 10000 points sampled randomly in the logarithmic starting position and the linear starting time. Core-dominated planets with an ice or water fraction of less than $25 \%$ are marked in yellow, core-dominated planets with an ice or water fraction between $25 \%$ and $50 \%$ are marked in blue and gas-dominated planets are marked in red. The water ice line is indicated by a dotted line.

random points in each growth map using a logarithmic distribution of starting points and a linear distribution of starting times. We also calculated the compositions of the planetary cores, by assuming that half of the mass of the pebbles is in the form of water ice outside of the water ice line. The water ice line is situated at around $0.45 \mathrm{AU}$ in our simulations due to the fact that we do not include viscous heating. The population synthesis demonstrates a clear dichotomy between those planets that reach the pebble isolation mass and undergo rapid gas accretion and those that stay below the pebble isolation mass. The runaway nature of early gas accretion leaves the region between 10 and 100 Earth masses relatively empty (this is known as the planet desert; see Ida \& Lin 2004).

Our pebble-accretion population-synthesis plots appear qualitatively similar to those presented in Bitsch \& Johansen (2017) and Ndugu et al. (2018). We refer to those two papers for comparisons to the observed exoplanet populations. One notable difference is the relatively fewer gas giants that reach the inner disc edge in our simulations. This is mainly an effect of the new planetary migration prescription that we explore here. Comparing our results to Brügger et al. (2018, who used a correction to the pebble sizes and fluxes of Bitsch et al. 2015a, resulting in very low pebble fluxes), we find very similar synthetic exoplanet populations, although the small-pebble model that we advocate here requires much lower pebble fluxes (and hence metallicities) to trigger the formation of gas giants in cold orbits. Chambers (2018) presented a comprehensive suite of population-synthesis calculations and used the observed exoplanet populations to pin down the best set of model input parameters for the protoplanetary disc and the pebble sizes. He found best fits to the data for low diffusion coefficients $\left(\sim 10^{-5}\right)$, which can be understood because of the positive effect of lowering the diffusion coefficient in the initial 3D stage of pebble accretion (we discuss this point further in Sect. 3.8).

Lin et al. (2018) considered planet formation in an $\alpha$-disc model with an evolving gas and pebble component. They studied a wide range of Stokes numbers $(S t)$ and disc viscosities $(\alpha)$ and found that it is mainly possible to form gas-giant planets when $S t>\alpha$. Smaller Stokes numbers maintain the global metallicity, as we also show in Eq. (14), but protoplanets accreting small pebbles experience a reduced pebble accretion rate both in the 2D and 3D Hill accretion branch. Lin et al. (2018) also reported that their synthetic planets either remain sub-Earth mass or "explode" to form gas giants. We recover this result in our own growth maps when considering only protoplanets started at $t=0$. Looking at the top-left panel of Fig. 5, we see that seeds starting at $t=0$ at distances of up to $15 \mathrm{AU}$ from the star migrate to the inner edge of the disc where they are stuck at a few Earth masses, while the seeds starting between 15 and 35 AU grow to warm and cold gas giants. Seeds starting even further out experience long growth timescales and therefore only some minor growth in situ. In our work we keep the actual time that it takes to form cores of $M=0.01 M_{\mathrm{E}}$ as a free parameter in our growth maps; the actual time that it takes to grow from planetesimals to such protoplanets is largely unknown and may be determined by the interplay between pebble accretion in the inefficient Bondi branch and mutual planetesimal-planetesimal collisions (Johansen \& Lambrechts 2017).

Super-Earths with final orbits within the water ice line in Fig. 7 transition from dry to water-rich at a mass of approximately one Earth mass, as more massive migrators accrete a large fraction of their mass outside of the water ice line. This composition appears to be in contrast with the inferred rocky compositions of super-Earths (Owen \& Wu 2017). However, we have neglected in this paper the viscous heating that would increase the disc aspect ratio $H / r$ in the inner regions of protoplanetary discs. An increase in the disc aspect ratio leads to lower migration rates in the inner protoplanetary disc and hence slows down the icy protoplanets that penetrate from beyond the ice line. The outwards migration resulting from such viscous heating will furthermore block the inwards flow of icy protoplanets of a certain mass range (Bitsch \& Johansen 2016) - we plan to include this effect in future studies using our model with a small Stokes number.

In Fig. 8 we show a population-synthesis calculation on a model where the temperature at $1 \mathrm{AU}$ is set to the higher value of $T_{1}=280 \mathrm{~K}$, corresponding to a sound speed of $c_{\mathrm{s} 1}=9.9 \times$ $10^{2} \mathrm{~m} \mathrm{~s}^{-1}$. This temperature profile corresponds to the Minimum Mass Solar Nebula model of Hayashi (1981). Through Eq. (23) this increase in sound speed leads to a larger maximum mass, due to the slower migration speed at an increased $H / r$. The result is 


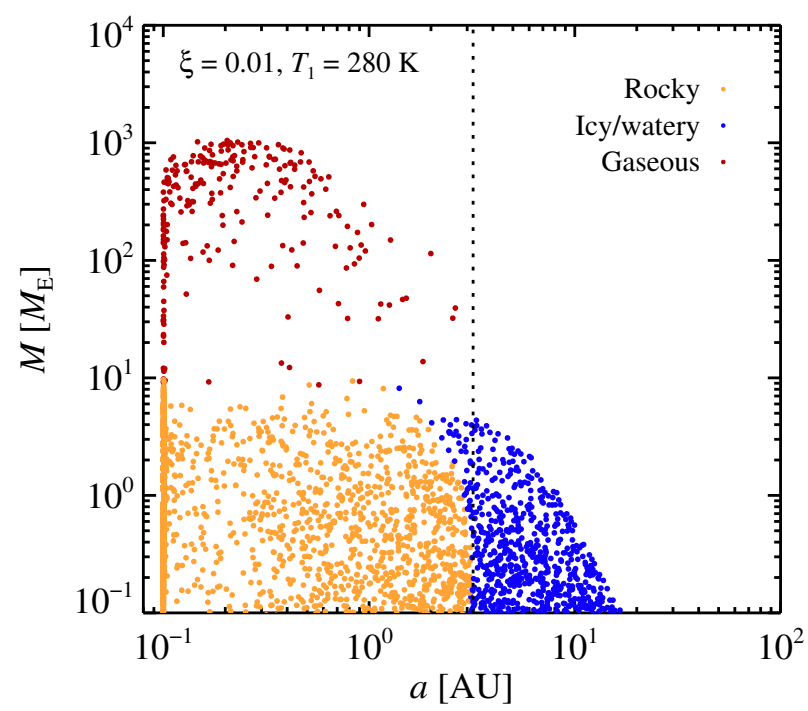

Fig. 8. Population synthesis plot for $\xi=0.01$ and a temperature of $T_{1}=280 \mathrm{~K}$ at $r=1 \mathrm{AU}$. The larger aspect ratio $H / r$, compared to Fig. 7 , slows down type I migration to a degree that icy protoplanets formed beyond the water ice line either stay small or grow into gas giant cores. This yields mainly dry super-Earths interior of the water ice line, in agreement with constraints from exoplanet observations (Owen \& Wu 2017). However, the increased gas scale-height also slows down both pebble accretion and gas accretion, resulting in fewer gas giants in the outer regions of the protoplanetary disc.

that terrestrial planets and super-Earths inside the water ice line are now mainly rocky. Icy protoplanets formed outside of the ice line either stay small, if they form late, or grow to gas giants.

\subsection{Different temporal accretion profiles}

We have so far analysed planet formation in a protoplanetary disc that evolves its accretion rate from $10^{-7}$ to $10^{-8} M_{\odot} \mathrm{yr}^{-1}$ over 3 Myr. Using $\alpha=0.01$ and our adapted temperature profile, such a temporal evolution implies that the initial disc size was $R_{1}=90.8 \mathrm{AU}$ and contained a mass of $0.12 M_{\odot}$ of gas and $400 M_{\mathrm{E}}$ of solids at a metallicity of $Z=0.01$. These values are similar to what is inferred for the iconic protoplanetary disc around HL Tau (Carrasco-González et al. 2016); such massive discs are common around young Class 0 and Class I objects (Tychoniec et al. 2018). However, surveys of more evolved protoplanetary discs with ages beyond approximately 1 Myr reveal typical dust masses of between 1 and $100 M_{\mathrm{E}}$ around solar-mass stars (Ansdell et al. 2017). Our nominal model with an accretion rate of $10^{-8} M_{\odot} \mathrm{yr}^{-1}$ at $3 \mathrm{Myr}$ contains $200 M_{\mathrm{E}}$ of solids at $1 \mathrm{Myr}$ and $30 M_{\mathrm{E}}$ at $3 \mathrm{Myr}$, that is towards the high end of the survey results presented in Ansdell et al. (2017).

The temporal decline of the gas-accretion rate in the $\alpha$-disc model is set by the viscous accretion timescale over the initial disc size (Hartmann et al. 1998). Fixing the global viscosity coefficient at $\alpha=0.01$, we find for our temperature profile that protoplanetary discs starting with an accretion rate of $10^{-7} M_{\odot} \mathrm{yr}^{-1}$ and reaching $5 \times 10^{-9} M_{\odot} \mathrm{yr}^{-1}$, $2 \times 10^{-9} M_{\odot} \mathrm{yr}^{-1}$ and $1 \times 10^{-9} M_{\odot} \mathrm{yr}^{-1}$ after $3 \mathrm{Myr}$ have initial sizes of $R_{1}=51.3,24.3$, and 15.0 AU, respectively. The initial masses of these discs are $0.074 M_{\odot}, 0.039 M_{\odot}$, and $0.026 M_{\odot}$, respectively.

The resulting growth maps for a fixed pebble-to-gas flux ratio of $\xi=0.02$ are shown in Fig. 9. Decreasing the initial disc size and mass leads to a significant decrease in the parameter space for forming gas-giant planets. Hence both the initial disc size and the metallicity are important factors in determining whether a protoplanetary disc will be able to form gas-giant planets. Manara et al. (2018) analysed the pebble masses in protoplanetary discs around young stars in the Lupus region, with ages between 1 and $3 \mathrm{Myr}$, and concluded that these masses are too low to explain the statistics of the observed exoplanet populations. They speculate that protoplanets must grow even earlier in the lifetime of the protoplanetary disc while there is still plenty of pebbles to accrete. Our results agree with this picture: planetary growth is very inefficient in the first million years and will proceed even in protoplanetary discs of initial sizes down to a few tens of astronomical units.

\subsection{Varying the model parameters}

In Fig. 10 we show the results of varying the parameters of our model, to probe how sensitive planet formation is to the physical properties of pebble growth, disc turbulence, and gap formation. All growth maps were calculated for $\xi=0.02$, as this pebble-to-gas flux ratio shows a large dependence on the physical parameters and thus makes differences clear.

The top row of Fig. 10 shows the result of varying the Stokes number. For larger Stokes number $S t=0.03$ the parameter space for forming gas-giant planets increases relative to the nominal case with $S t=0.01$. This increase in Stokes number affects the initial 3D pebble accretion stage most positively, since the accretion rate in the 3D Hill regime is proportional to the Stokes number. Lowering the Stokes number to $S t=0.003$, on the other hand, has a devastating effect on the ability of the protoplaetary disc to form gas giants. The protoplanets are stuck at 3D growth below one Earth mass, unless they start very early.

In the middle row of Fig. 10, we increase the turbulent viscosity (and the turbulent diffusion coefficient $\delta$ ) to $\alpha_{\mathrm{v}}=3 \times 10^{-4}$ and $\alpha_{\mathrm{v}}=10^{-3}$. Pinte et al. (2016) inferred $\delta \sim 3 \times 10^{-4}$ from measurements of the scale height of the pebble layer. The translation from particle layer scale height to turbulent diffusion coefficient nevertheless depends on the assumed Stokes number. The high value of $\alpha_{\mathrm{v}}$ (and hence of $\delta$ ) pushes planet formation to earlier times than for our nominal case of $\alpha_{\mathrm{v}}=10^{-4}$ used in Fig. 5.

Finally, we show in the bottom row of Fig. 10 two additional experiments with gap formation. In the left panel we use the $50 \%$ gap mass from Kanagawa et al. (2018) and the pebble isolation mass from the 3D simulations of Bitsch et al. (2018b). Taking these measurements at face value, it is clear from Fig. 3 that the pebble isolation mass is actually higher than the $50 \%$ gap mass at $\alpha_{\mathrm{v}}=10^{-4}$. Since the $50 \%$ gap mass is only determined by the relative height of the gap, while the pebble isolation mass is set by both the gap height and shape, it could be that gap edge instabilities would broaden the gap in such a way that the pebble isolation mass comes after the $50 \%$ gap mass. The result is a major reduction in planetary migration. The final planetary mass is also reduced; this is a consequence of the reduced migration, since the gas-accretion rates are higher in the inner regions of the protoplanetary disc where $H / r$ is low, according to our adopted gas-accretion model from Tanigawa \& Tanaka (2016). In the right panel we assume instead that the $50 \%$ gap mass is given by Kanagawa et al. (2018), while the pebble isolation mass is 2.3 times lower. Hence the pebble isolation mass is now only of the order of one Earth mass, resulting in both reduced gas-accretion rates (due to the small core mass) and reduced migration (due to the small gap mass). 


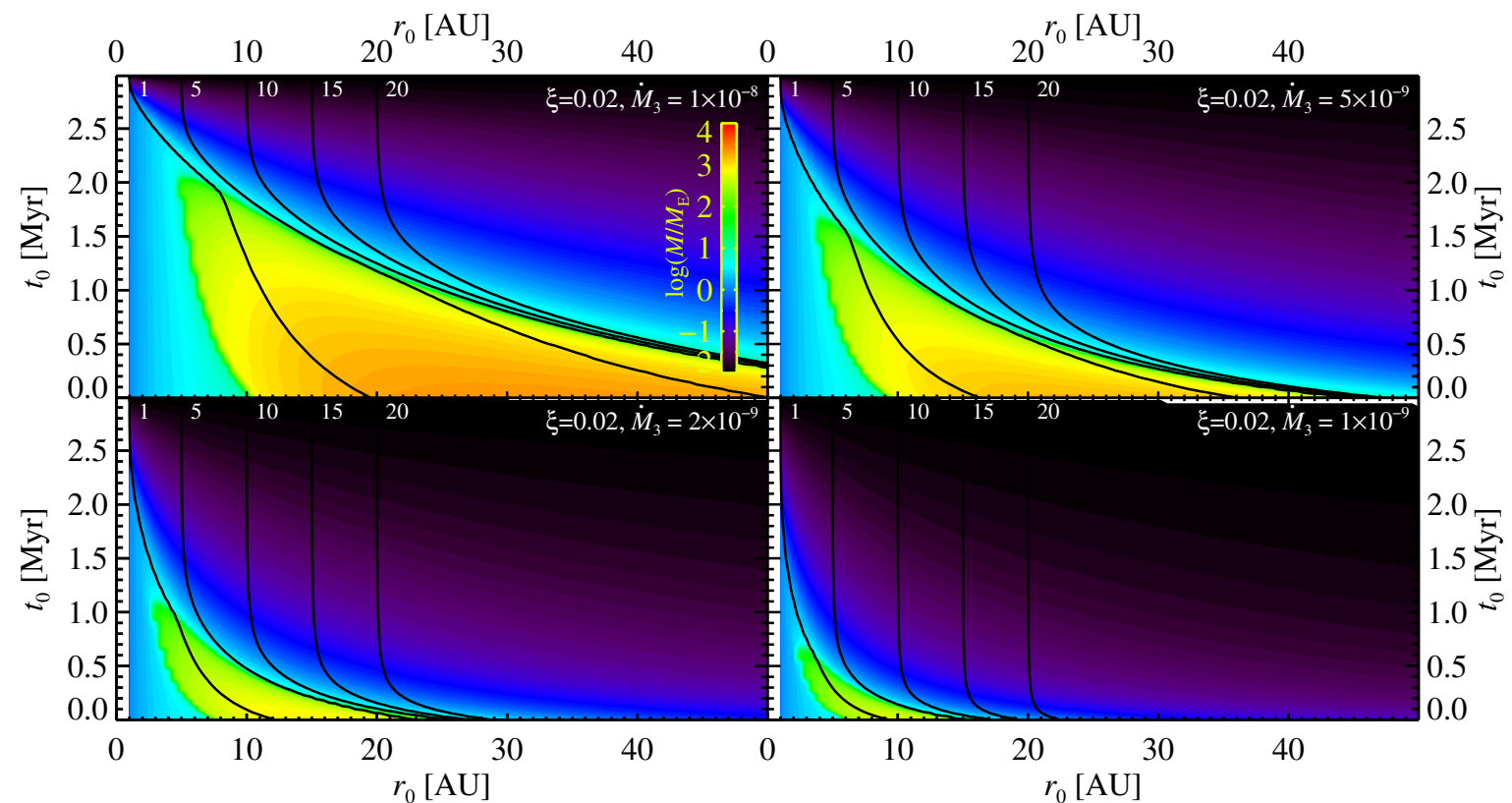

Fig. 9. Growth maps for four values of $\dot{M}_{3}$, the mass-accretion rate after $3 \mathrm{Myr}$, with fixed pebble-to-gas flux ratio $\xi=0.02$. The initial accretion rate is $10^{-7} M_{\odot} \mathrm{yr}^{-1}$ in all models. Increasing the steepness of the temporal accretion rate profile, obtained here by lowering the initial disc size, decreases the region for forming gas-giant planets.

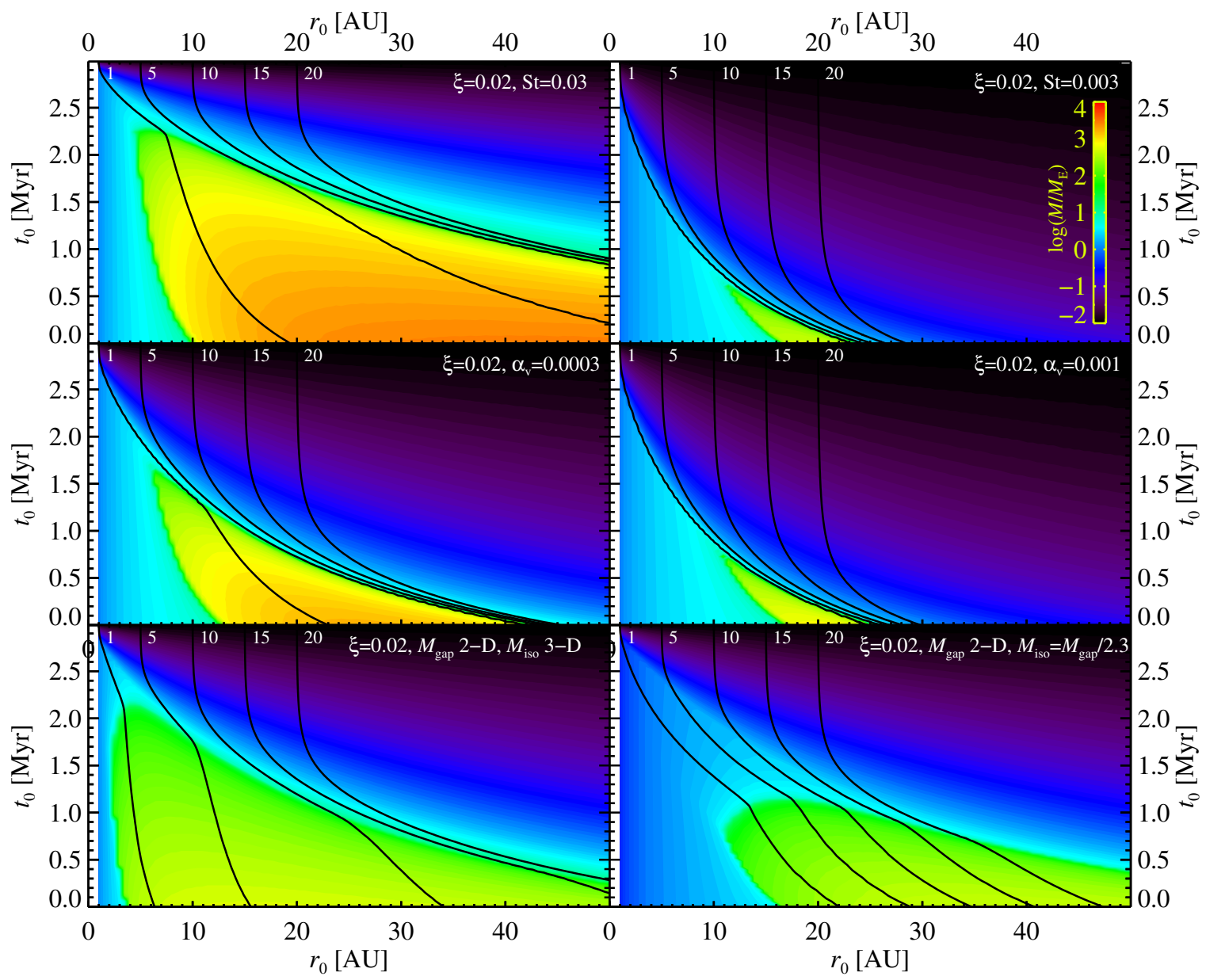

Fig. 10. Growth maps for six variations of our nominal model. Top panels: higher and lower Stokes number, $S t=0.03$ and $S t=0.003$. The Stokes number is an important factor in the pebble-accretion rate, particularly in the initial stage of 3D pebble accretion. Middle panels: higher turbulent viscosity (and turbulent diffusion), $\alpha_{\mathrm{v}}=0.0003$ and $\alpha_{\mathrm{v}}=0.001$. Planet formation is pushed to earlier times as the turbulent viscosity is increased. Bottom panels: variations of the gap formation prescriptions, either using the 50\% gap mass from the 2D simulations of Kanagawa et al. (2018) and the pebble isolation mass from the 3D simulations of Bitsch et al. (2018b; bottom left panel) or using the $50 \%$ gap mass from the 2D simulations of Kanagawa et al. (2018) and a pebble isolation mass that is less than that value by a factor of 2.3. Both result in decreased migration and decreased gas accretion. 


\section{Summary and discussion}

In this paper we have addressed the question of how planetary growth is able to outperform migration. Type-I migration likely operates at its full strength in the outer regions of the protoplanetary disc where planetary cores grow to form gas giants and ice giants, in contrast to the viscously heated inner regions of the protoplanetary disc where the positive corotation torques slow down or reverse the migration of super-Earth cores (Bitsch \& Johansen 2016; Brasser et al. 2017).

We derived analytical growth tracks for a protoplanet undergoing pebble accretion while migrating towards the star, under the assumptions that the pebble Stokes number is a constant both in time and in space. Furthermore, we have shown that the pebble mid-plane layer is sufficiently thin for pebble accretion to take place in $2 \mathrm{D}$, and that the mass flux of pebbles is a constant ratio of the radial gas flow. We used these analytical expressions to derive the radial location where the growing core reaches the pebble isolation mass. Our analytical growth tracks demonstrate that protoplanets undergo substantial migration during their growth towards the pebble isolation mass and that the location where the pebble isolation mass is reached increases as the square root of the starting location.

An important new ingredient in our model, compared to previous studies of pebble accretion and migration (Bitsch et al. 2015a; Matsumura et al. 2017), is the reduction of the migration torque due to the growing planetary gap (Kanagawa et al. 2018). We identify here the close connection between the $50 \%$ gap mass, $M_{\text {gap }}$, of Kanagawa et al. (2018) and the pebble isolation mass, $M_{\text {iso }}$, of Lambrechts et al. (2014). From numerical simulations of low-mass planets embedded in a 1D model of a protoplanetary disc, we infer that $M_{\text {gap }} \approx 2.3 M_{\text {iso }}$. Reaching first the pebble isolation mass and slightly later the gap transition mass thus signifies three important events in the growth of a protoplanet: (a) the end of the accretion of pebbles, (b) the beginning of gas contraction and (c) the transition to a slow-down in the migration caused by the deepening gap. Gas accretion can therefore take place over just a few astronomical units of migration. We emphasise that the connection between the $50 \%$ gap mass and the pebble isolation mass is derived here from 1D simulations and that dedicated 2D and 3D simulations will be needed in the future to decipher whether or not this simplified relationship holds. At low viscosity $\left(\alpha<10^{-3}\right)$ our measured pebble isolation mass is significantly lower than the measurements from 3D simulations (Bitsch et al. 2018b); this may be either due to gap-edge instabilities that are not captured in the 1D approach (Hallam \& Paardekooper 2017) or due to the limited simulation time that can be afforded in $3 \mathrm{D}$.

We have demonstrated that protoplanets can grow to gasgiant planets in models where pebbles are relatively small, with a Stokes number of 0.01 corresponding to approximately millimetre-sized particles in the inner regions of the protoplanetary disc, and that the pebble-to-gas flux ratio is in the nominal range $\xi=0.01-0.02$, in contrast to the models of Bitsch et al. (2015a, 2018a), and Brügger et al. (2018) where pebbles were allowed to grow to the radial drift barrier. The radial drift of the small pebbles considered in this work is dominated by the advection with the gas accretion flow - and the gas and pebble components of the protoplanetary disc therefore drain onto the central star on an approximately similar timescale. This way we avoid the radial drift problem where large pebbles drift out of the protoplanetary disc on a timescale that is much shorter than the gas-accretion timescale (Brauer et al. 2007).
Our results show that the cores of cold gas giants ending in orbits of 5-10 AU must generally start their assembly from planetesimals forming in the 15-30 AU region. This raises the question of why planetesimals forming at such distances would be the seeds of the gas giants. The water ice line has been demonstrated to be a preferred location for the formation of planetesimals (Ros \& Johansen 2013; Ida \& Guillot 2016; Schoonenberg \& Ormel 2017; Drążkowska \& Alibert 2017). If the ice lines of more volatile species such as CO (Qi et al. 2013) are equally prone to forming planetesimals, then this may explain why the cores of the gas-giants in the solar system started as seeds far away from the Sun and subsequently migrated to their current orbits.

Acknowledgements. The authors thank the anonymous referee whose report helped us to improve the original manuscript. We would furthermore like to thank Hidekazu Tanaka, Kazuhiro Kanagawa, Bertram Bitsch and Christoph Mordasini for inspiring discussions. A.J. is grateful to the Earth-Life Science Institute (ELSI) at Tokyo Institute of Technology for hosting his research visit during March 2018. A.J. further thanks the Swedish Research Council (grant 2014-5775), the Knut and Alice Wallenberg Foundation (grants 2012.0150, 2014.0017) and the European Research Council (ERC Consolidator Grant 724687-PLANETESYS) for research support. S.I. is supported by JSPS grant $15 \mathrm{H} 02065$ and MEXT grant $18 \mathrm{H} 05438$.

\section{References}

Alexander, R. D., \& Pascucci, I. 2012, MNRAS, 422, L82

Ansdell, M., Williams, J. P., Manara, C. F., et al. 2017, AJ, 153, 240

Armitage, P. J., Simon, J. B., \& Martin, R. G. 2013, ApJ, 778, L14

Ataiee, S., Baruteau, C., Alibert, Y., \& Benz, W. 2018, A\&A, 615, A110

Bai, X.-N. 2015, ApJ, 798, 84

Bai, X.-N., \& Stone, J. M. 2010, ApJ, 722, 1437

Bai, X.-N., \& Stone, J. M. 2013, ApJ, 769, 76

Batalha, N. M., Rowe, J. F., Bryson, S. T., et al. 2013, ApJS, 204, 24

Benítez-Llambay, P., \& Pessah, M. E. 2018, ApJ, 855, L28

Benítez-Llambay, P., Masset, F., Koenigsberger, G., \& Szulágyi, J. 2015, Nature, 520,63

Béthune, W., Lesur, G., \& Ferreira, J. 2017, A\&A, 600, A75

Birnstiel, T., Klahr, H., \& Ercolano, B. 2012, A\&A, 539, A148

Bitsch, B., \& Johansen, A. 2016, A\&A, 590, A101

Bitsch, B., \& Johansen, A. 2017, Astrophys. Space Sci. Lib., 445, 339

Bitsch, B., Morbidelli, A., Lega, E., Kretke, K., \& Crida, A. 2014, A\&A, 570, A75

Bitsch, B., Lambrechts, M., \& Johansen, A. 2015a, A\&A, 582, A112

Bitsch, B., Johansen, A., Lambrechts, M., \& Morbidelli, A. 2015b, A\&A, 575, A28

Bitsch, B., Lambrechts, M., \& Johansen, A. 2018a, A\&A, 609, C2

Bitsch, B., Morbidelli, A., Johansen, A., et al. 2018b, A\&A, 612, A30

Brasser, R., Bitsch, B., \& Matsumura, S. 2017, AJ, 153, 222

Brauer, F., Dullemond, C. P., Johansen, A., et al. 2007, A\&A, 469, 1169

Brauer, F., Dullemond, C. P., \& Henning, T. 2008, A\&A, 480, 859

Brügger, N., Alibert, Y., Ataiee, S., \& Benz, W. 2018, A\&A, 619, A174

Carrasco-González, C., Henning, T., Chandler, C. J., et al. 2016, ApJ, 821, L16

Chambers, J. 2018, ApJ, 865, 30

Chiang, E., \& Youdin, A. N. 2010, Ann. Rev. Earth Planet. Sci., 38, 493

D'Angelo, G., \& Bodenheimer, P. 2013, ApJ, 778, 77

D'Angelo, G., \& Lubow, S. H. 2010, ApJ, 724, 730

Drążkowska, J., \& Alibert, Y. 2017, A\&A, 608, A92

Drążkowska, J., Alibert, Y., \& Moore, B. 2016, A\&A, 594, A105

Duffell, P. C., \& MacFadyen, A. I. 2013, ApJ, 769, 41

Dürmann, C., \& Kley, W. 2015, A\&A, 574, A52

Ercolano, B., \& Rosotti, G. 2015, MNRAS, 450, 3008

Fung, J., \& Chiang, E. 2016, ApJ, 832, 105

Fung, J., \& Lee, E. J. 2018, ApJ, 859, 126

Fung, J., Shi, J.-M., \& Chiang, E. 2014, ApJ, 782, 88

Goldreich, P., \& Tremaine, S. 1980, ApJ, 241, 425

Gomes, R., Levison, H. F., Tsiganis, K., \& Morbidelli, A. 2005, Nature, 435, 466

Gonzalez, J.-F., Laibe, G., \& Maddison, S. T. 2017, MNRAS, 467, 1984

Gorti, U., Hollenbach, D., \& Dullemond, C. P. 2015, ApJ, 804, 29

Hallam, P. D., \& Paardekooper, S.-J. 2017, MNRAS, 469, 3813

Hartmann, L., Calvet, N., Gullbring, E., \& D’Alessio, P. 1998, ApJ, 495, 385

Hartmann, L., Herczeg, G., \& Calvet, N. 2016, ARA\&A, 54, 135 
Hasegawa, Y., Okuzumi, S., Flock, M., \& Turner, N. J. 2017, ApJ, 845, 31

Hayashi, C. 1981, Prog. Theor. Phys. Suppl., 70, 35

Huang, J., Andrews, S. M., Cleeves, L. I., et al. 2018, ApJ, 852, 122

Ida, S., \& Guillot, T. 2016, A\&A, 596, L3

Ida, S., \& Lin, D. N. C. 2004, ApJ, 604, 388

Ida, S., Guillot, T., \& Morbidelli, A. 2016, A\&A, 591, A72

Ida, S., Tanaka, H., Johansen, A., Kanagawa, K., \& Tanigawa, T. 2018, ApJ, 864, 77

Ikoma, M., Nakazawa, K., \& Emori, H. 2000, ApJ, 537, 1013

Izidoro, A., Morbidelli, A., Raymond, S. N., Hersant, F., \& Pierens, A. 2015, A\&A, 582, A99

Izidoro, A., Ogihara, M., Raymond, S. N., et al. 2017, MNRAS, 470, 1750

Johansen, A., \& Klahr, H. 2005, ApJ, 634, 1353

Johansen, A., \& Lambrechts, M. 2017, Ann. Rev. Earth Planet. Sci., 45, 359

Johansen, A., Youdin, A., \& Klahr, H. 2009a, ApJ, 697, 1269

Johansen, A., Youdin, A. N., \& Mac Low, M.-M. 2009b, ApJ, 704, L75

Johansen, A., Blum, J., Tanaka, H., et al. 2014, Protostars and Planets VI (Tucson, AZ: University of Arizona Press), 547

Johansen, A., Mac Low, M.-M., Lacerda, P., \& Bizzarro, M. 2015, Sci. Adv., 1, 1500109

Kanagawa, K. D., Tanaka, H., Muto, T., Tanigawa, T., \& Takeuchi, T. 2015, MNRAS, 448, 994

Kanagawa, K. D., Tanaka, H., \& Szuszkiewicz, E. 2018, ApJ, 861, 140

Klahr, H., \& Kley, W. 2006, A\&A, 445, 747

Lambrechts, M., \& Johansen, A. 2012, A\&A, 544, A32

Lambrechts, M., \& Johansen, A. 2014, A\&A, 572, A107

Lambrechts, M., \& Lega, E. 2017, A\&A, 606, A146

Lambrechts, M., Johansen, A., \& Morbidelli, A. 2014, A\&A, 572, A35

Lambrechts, M., Lega, E., Morbidelli, A., \& Nelson, R. 2018, A\&A, submitted

Lee, E. J., \& Chiang, E. 2015, ApJ, 811, 41

Lee, E. J., \& Chiang, E. 2016, ApJ, 817, 90

Lee, E. J., \& Chiang, E. 2017, ApJ, 842, 40

Li, H., Lubow, S. H., Li, S., \& Lin, D. N. C. 2009, ApJ, 690, L52

Lin, D. N. C., \& Papaloizou, J. 1979, MNRAS, 186, 799

Lin, J. W., Lee, E. J., \& Chiang, E. 2018, MNRAS, 480, 4338

Liu, B., \& Ormel, C. W. 2018, A\&A, 615, A138

Liu, B., Ormel, C. W., \& Lin, D. N. C. 2017, A\&A, 601, A15

Lodders, K. 2004, ApJ, 611, 587

Lubow, S. H., \& D’Angelo, G. 2006, ApJ, 641, 526

Lyra, W., Johansen, A., Klahr, H., \& Piskunov, N. 2008, A\&A, 479, 883

Lyra, W., Johansen, A., Klahr, H., \& Piskunov, N. 2009, A\&A, 493, 1125

Lyra, W., Paardekooper, S.-J., \& Mac Low, M.-M. 2010, ApJ, 715, L68

Madhusudhan, N., Bitsch, B., Johansen, A., \& Eriksson, L. 2017, MNRAS, 469, 4102

Manara, C. F., Morbidelli, A., \& Guillot, T. 2018, A\&A, 618, L3

Matsumura, S., Brasser, R., \& Ida, S. 2017, A\&A, 607, A67
Menu, J., van Boekel, R., Henning, T., et al. 2014, A\&A, 564, A93

Morbidelli, A., Lambrechts, M., Jacobson, S., \& Bitsch, B. 2015, Icarus, 258, 418

Mordasini, C. 2014, A\&A, 572, A118

Mulders, G. D., \& Dominik, C. 2012, A\&A, 539, A9

Muñoz Caro, G. M., \& Schutte, W. A. 2003, A\&A, 412, 121

Musiolik, G., Teiser, J., Jankowski, T., \& Wurm, G. 2016a, ApJ, 818, 16

Musiolik, G., Teiser, J., Jankowski, T., \& Wurm, G. 2016b, ApJ, 827, 63

Ndugu, N., Bitsch, B., \& Jurua, E. 2018, MNRAS, 474, 886

Okuzumi, S., Tanaka, H., Kobayashi, H., \& Wada, K. 2012, ApJ, 752, 106

Ormel, C. W., \& Cuzzi, J. N. 2007, A\&A, 466, 413

Ormel, C. W., \& Klahr, H. H. 2010, A\&A, 520, A43

Ormel, C. W., Liu, B., \& Schoonenberg, D. 2017, A\&A, 604, A1

Owen, J. E., \& Wu, Y. 2017, ApJ, 847, 29

Paardekooper, S.-J., Baruteau, C., Crida, A., \& Kley, W. 2010, MNRAS, 401, 1950

Pérez, L. M., Carpenter, J. M., Chandler, C. J., et al. 2012, ApJ, 760, L17

Pinte, C., Dent, W. R. F., Ménard, F., et al. 2016, ApJ, 816, 25

Piso, A.-M. A., \& Youdin, A. N. 2014, ApJ, 786, 21

Popovas, A., Nordlund, A., Ramsey, J. P., \& Ormel, C. W. 2018, MNRAS, 479, 5136

Pringle, J. E. 1981, ARA\&A, 19, 137

Qi, C., Öberg, K. I., Wilner, D. J., et al. 2013, Science, 341, 630

Rafikov, R. R. 2002, ApJ, 572, 566

Ros, K., \& Johansen, A. 2013, A\&A, 552, A137

Safronov, V. S. 1969, Evoliutsiia doplanetnogo oblaka (English transl.: Evolution of the Protoplanetary Cloud and Formation of Earth and the Planets, NASA Tech. Transl. F-677, Jerusalem: Israel Sci. Transl. 1972)

Schoonenberg, D., \& Ormel, C. W. 2017, A\&A, 602, A21

Shiraishi, M., \& Ida, S. 2008, ApJ, 684, 1416

Tanaka, H., \& Ida, S. 1999, Icarus, 139, 350

Tanaka, H., Takeuchi, T., \& Ward, W. R. 2002, ApJ, 565, 1257

Tanigawa, T., \& Tanaka, H. 2016, ApJ, 823, 48

Testi, L., Natta, A., Shepherd, D. S., \& Wilner, D. J. 2003, A\&A, 403, 323

Trotta, F., Testi, L., Natta, A., Isella, A., \& Ricci, L. 2013, A\&A, 558, A64

Tychoniec, Ł., Tobin, J. J., Karska, A., et al. 2018, ApJS, 238, 19

van Boekel, R., Henning, T., Menu, J., et al. 2017, ApJ, 837, 132

Venturini, J., \& Helled, R. 2017, ApJ, 848, 95

Wahl, S. M., Hubbard, W. B., Militzer, B., et al. 2017, Geophys. Res. Lett., 44, 4649

Wilner, D. J., D’Alessio, P., Calvet, N., Claussen, M. J., \& Hartmann, L. 2005, ApJ, 626, L109

Weidenschilling, S. J. 1977, MNRAS, 180, 57

Youdin, A., \& Goodman, J. 2005, ApJ, 620, 459

Zsom, A., Ormel, C. W., Güttler, C., Blum, J., \& Dullemond, C. P. 2010, A\&A, 513, A57 


\section{Appendix A: Life-time of the pebble component of protoplanetary discs}

Protoplanetary discs are efficient at converting dust to pebbles due to their high gas densities and weak turbulence. Testi et al. (2003) and Wilner et al. (2005) inferred the presence of cmsized pebbles in protoplanetary discs around CQ Tau and TW Hya, respectively, from the spectral energy distribution. Many other protoplanetary discs have been resolved at $\mathrm{cm}$ wavelengths, including AS 209 (Pérez et al. 2012), CQ Tau (Trotta et al. 2013) and HL Tau (Carrasco-González et al. 2016), to reveal mm-sized pebbles in the outer parts of the discs and $\mathrm{cm}$-sized pebbles in the inner regions. Ansdell et al. (2017) presented extensive survey results on dust masses of protoplanetary discs observed with ALMA and found generally 1-100 Earth masses of mm-sized pebbles remaining in relatively evolved discs around solar-type stars with ages in excess of $1 \mathrm{Myr}$.

The presence of pebbles in protoplanetary is difficult to reconcile with the short drift time-scales of pebbles (Brauer et al. 2007). Lambrechts \& Johansen (2014) proposed that the delayed growth from dust to pebbles in the low-density environment of the protoplanetary disc beyond 100 AU provides a mechanism to maintain a long life-time of the pebble disc. The mass flux of pebbles is determined not by the radial drift of the large pebbles close to the star, but by the timescale to grow to drifting sizes in the outer disc. However, their model did not incorporate the exponentially tapered outer regions of the protoplanetary disc, which form as a natural consequence of outwards angular momentum transport (Pringle 1981).

We explore here the evolution of drifting pebbles in an $\alpha$-model of a protoplanetary disc. We let the gas evolve according to the analytical viscous disc equations given in Hartmann et al. (1998) and simulate the motion of 20000 pebble superparticles, with the mass of each pebble swarm set to give an initial dust-to-gas ratio $Z=0.01$ everywhere. In Fig. A.1 we show the total gas and pebble masses (top panel) and mass accretion rates (bottom panel) for a disc that evolves from $\dot{M}=10^{-7} M_{\odot} \mathrm{yr}^{-1}$ to $\dot{M}=10^{-8} M_{\odot} \mathrm{yr}^{-1}$ over 3 Myr. We adopt $\alpha=10^{-2}$ for the disc evolution, so that the disc has an initial size of $R_{1}=90.8 \mathrm{AU}$. We use six models for the pebble sizes: (i) constant Stokes number $S t=0.001$, (ii) constant Stokes number $S t=0.01$, (iii) constant Stokes number $S t=0.1$, (iv) constant pebble size $R=1 \mathrm{~mm}$, (v) constant pebble size $R=1 \mathrm{~cm}$ and (vi) variable Stokes number $S t=0.01[r /(10 \mathrm{AU})]^{-1}$. The gas mass in these $\alpha$-disc models falls only slowly with time; this is due to the power-law nature of the temporal evolution of the accretion rate. Protoplanetary discs may need FUV photoevaporation to dissipate these viscously spreading outer regions (Gorti et al. 2015).

The simulations presented in Fig. A.1 show mm-sized pebbles, cm-sized pebbles, and pebbles with $S t=0.1$ to be quickly drained out of the protoplanetary disc, in much less than 1 Myr. The pebble fluxes are initially very high, with $\xi=\dot{M}_{\mathrm{p}} / \dot{M}_{\mathrm{g}}$ reaching values above 0.1 . While such mass fluxes could certainly lead to very high pebble-accretion rates, the rapid depletion of pebbles appears to be in conflict with observations of 1-100 $M_{\mathrm{E}}$ of pebbles in evolved protoplanetary discs around solar-mass stars (Ansdell et al. 2017).

The models with $S t=0.01, S t=0.001$ or power-law Stokes number are more successful at maintaining the pebbles for the life-time of the protoplanetary disc. Here the low drift rates of the small pebbles, as well as the outwards drift of pebbles in the viscously expanding outer regions of the protoplanetary disc, keep the pebble flux at a level of around $1 \%$ of the gas flux. The model
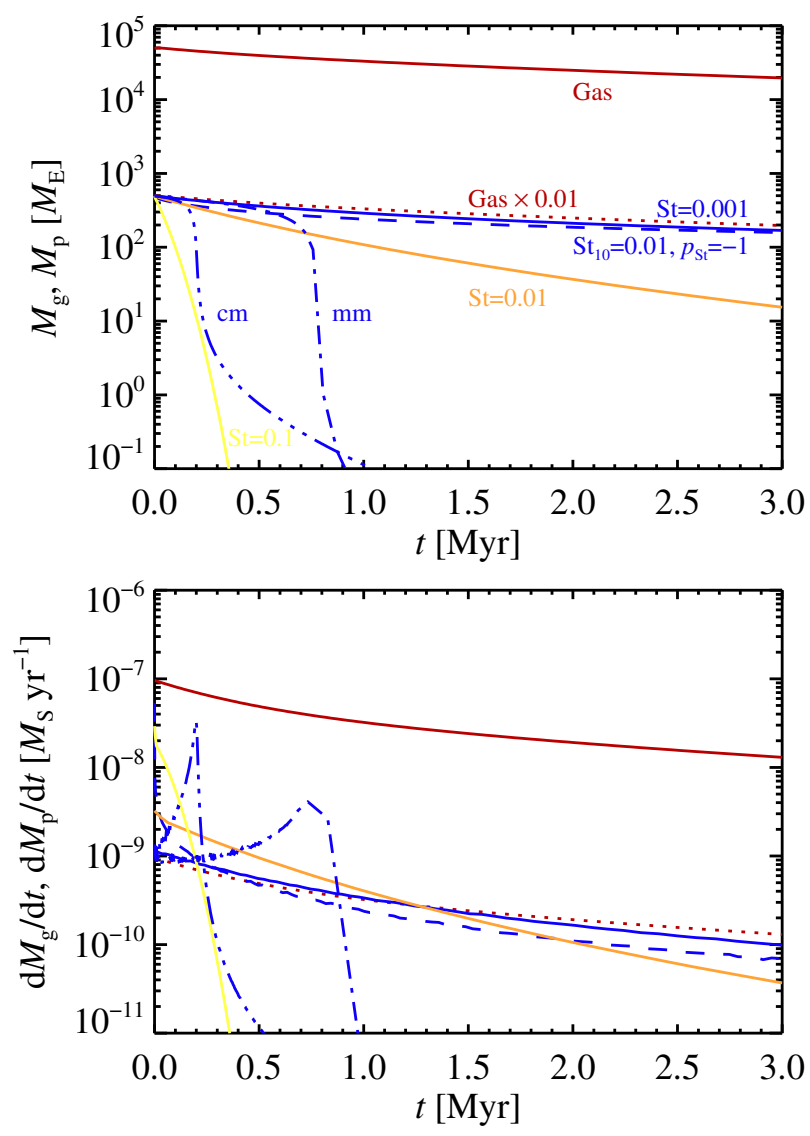

Fig. A.1. Gas and pebble masses as a function of time (top panel) and the mass accretion rates of gas and pebbles (bottom panel). The different pebble models are explained in the main text. Protoplanetary discs with large pebbles - of $\mathrm{mm}$ or $\mathrm{cm}$ sizes or with constant Stokes number of 0.1 - drain their pebbles out of the disc in much less than $1 \mathrm{Myr}$. The mass-accretion rates of such large pebbles is very high during this period. The models with a lower Stokes number are better able to maintain their pebbles for the entire life-time of the protoplanetary disc.

with $S t=0.01$ at $10 \mathrm{AU}$ and falling inversely proportionally to the distance could have its physical foundation in a turbulent $\alpha$ that increases in the outer disc, limiting the particle growth there (as proposed in Ida et al. 2016). For this model we also limited the smallest particle size to one micron.

In Fig. A.2 we show the gas and pebble evolution for an initial disc size of $24.3 \mathrm{AU}$. The initial disc mass is thus only $0.039 M_{\odot}$, towards the low end for the distribution of masses of embedded Class 0 and Class I objects (Tychoniec et al. 2018). Even small pebbles with $S t=0.01$ only survive for less than $1 \mathrm{Myr}$ in such a small disc. Tiny pebbles with $S t=0.001$ nevertheless follow the gas accretion profile closely, as does the model with a Stokes number that decreases with distance.

While the realism of the $\alpha$-model that we use here for the gas is debatable in light of modern disc-wind models for accretion (Bai \& Stone 2013; Béthune et al. 2017), overall we find that the presence of pebbles in protoplanetary discs for million years may be determined by the limited growth of particles in the outer regions of the protoplanetary disc that acts as a drift bottleneck. Such small and cold dust far from star would be hard to detect but may reveal its presence in scattered light images of protoplanetary discs that often extends to sizes much larger than at mm wavelengths (e.g. van Boekel et al. 2017). 

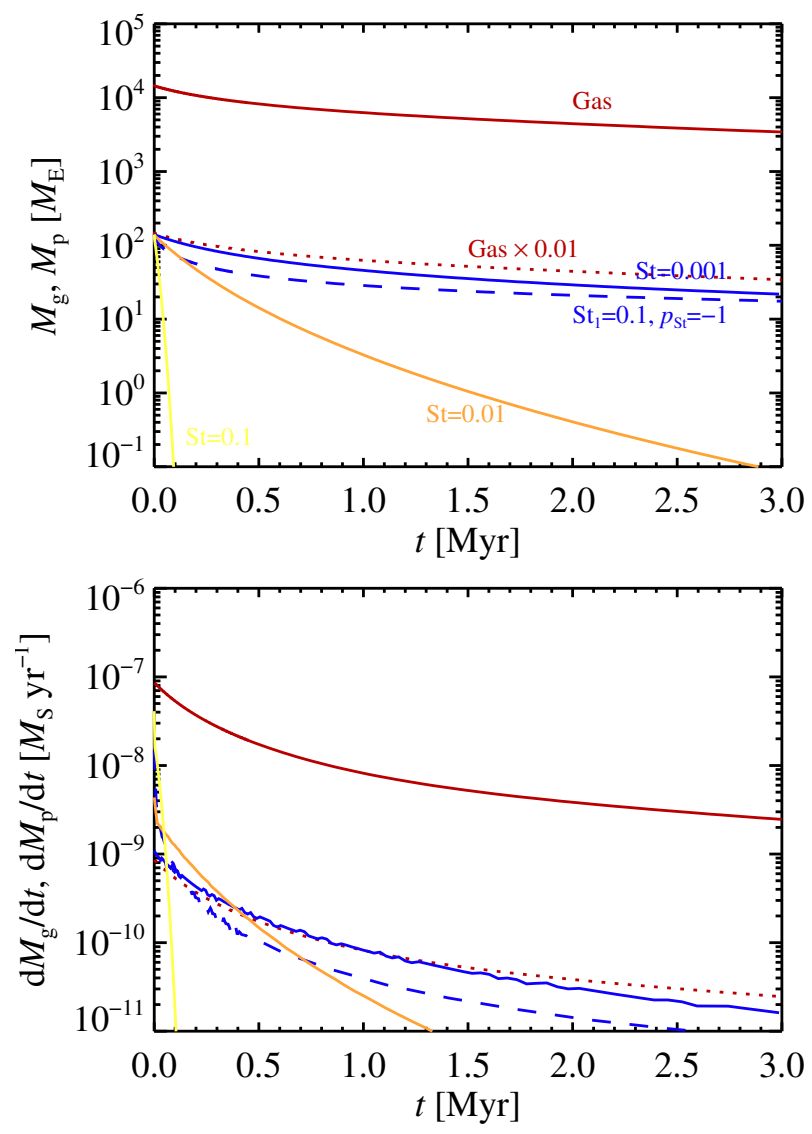

Fig. A.2. Gas and pebble masses (top panel) and accretion rates (bottom panel) for a disc of small initial size $R_{1}=24.3 \mathrm{AU}$ and initial mass $M=0.039 M_{\odot}$. Pebbles of $S t=0.01$ are drained from the disc within $1 \mathrm{Myr}$ due to the small initial disc size. However, smaller pebbles and pebbles with a radially declining Stokes number follow the temporal decay profile of the gas and are hence depleted on the same timescale as the gas.

\section{Appendix B: Analytical expression for the growth track time}

In this appendix, we derive the time associated with the analytical growth track derived in Sect. 2. We replace the pebble surface density $\Sigma_{\text {p }}$ in Eq. (1) by Eq. (13) and split out the specific dependence on $M$ and $r$ to obtain

$$
\begin{aligned}
\dot{M}= & 2\left(\frac{S t}{0.1}\right)^{2 / 3} G M_{\star}\left(3 M_{\star}\right)^{-2 / 3} \\
& \times \frac{\xi \dot{M}_{\mathrm{g}}(t)}{2 \pi[\chi S t+(3 / 2) \alpha] c_{\mathrm{s} 1}^{2} \mathrm{AU}^{\zeta}} M^{2 / 3} r^{\zeta-1} .
\end{aligned}
$$

We need now a model for the evolution of the protoplanetary disc. We use the standard $\alpha$-disc evolution model of Sect. 3. The turbulent viscosity follows the power-law

$v=\alpha c_{\mathrm{s}} H \propto r^{\gamma}$.

Here the power-law index $\gamma$ can be written as

$\gamma=(3 / 2)-\zeta$. 1998)

The gas accretion onto the star evolves as (Hartmann et al.

$\dot{M}_{\mathrm{g}}=\dot{M}_{\mathrm{g} 0} T^{-(5 / 2-\gamma) /(2-\gamma)}$, where the non-dimensional time is

$T=t / t_{\mathrm{s}}+1$,

the characteristic time is

$t_{\mathrm{s}}=\frac{1}{3(2-\gamma)^{2}} \frac{R_{1}^{2}}{v_{1}}$

and $R_{1}$ is the initial disc size and $v_{1}$ the viscosity at that location. We now insert the inverse solution for the growth track, $r(M)$, and separate the variables $M$ and $r$ to yield the equation

$$
\begin{aligned}
& r_{0}^{1-\zeta} M^{-2 / 3}\left(1-\frac{M^{4 / 3}-M_{0}^{4 / 3}}{M_{\max }^{4 / 3}-M_{0}^{4 / 3}}\right) \mathrm{d} M \\
& \quad=\frac{2(S t / 0.1)^{2 / 3} G M_{\star}\left(3 M_{\star}\right)^{-2 / 3} \xi \dot{M}_{\mathrm{g} 0}}{2 \pi[\chi S t+(3 / 2) \alpha] c_{\mathrm{s} 1}^{2} \mathrm{AU}^{\zeta}} T^{-(5 / 2-\gamma) /(2-\gamma)} t_{\mathrm{s}} \mathrm{d} T .
\end{aligned}
$$

We integrate both sides to yield

$$
\begin{aligned}
r_{0}^{1-\zeta}\left[\frac{-(3 / 5)\left(M^{5 / 3}-M_{0}^{5 / 3}\right)+3\left(M^{1 / 3}-M_{0}^{1 / 3}\right) M_{\max }^{4 / 3}}{M_{\max }^{4 / 3}-M_{0}^{4 / 3}}\right] \\
=\frac{2(S t / 0.1)^{2 / 3} G M_{\star}\left(3 M_{\star}\right)^{-2 / 3} \xi \dot{M}_{\mathrm{g} 0}}{2 \pi[\chi S t+(3 / 2) \alpha] c_{\mathrm{s} 1}^{2} \mathrm{AU}^{\zeta}} \\
\quad \times \frac{\left[T^{-(5 / 2-\gamma) /(2-\gamma)+1}-T_{0}^{-(5 / 2-\gamma) /(2-\gamma)+1}\right] t_{\mathrm{s}}}{-(5 / 2-\gamma) /(2-\gamma)+1} .
\end{aligned}
$$

We now divide the equation by the solution at $r=0$, $M\left(T_{\max }\right)=M_{\max }$, to give the simpler expression

$$
\begin{gathered}
\frac{-(3 / 5)\left(M^{5 / 3}-M_{0}^{5 / 3}\right)+3\left(M^{1 / 3}-M_{0}^{1 / 3}\right) M_{\max }^{4 / 3}}{-(3 / 5)\left(M_{\max }^{5 / 3}-M_{0}^{5 / 3}\right)+3\left(M_{\max }^{1 / 3}-M_{0}^{1 / 3}\right) M_{\max }^{4 / 3}} \\
=\frac{T^{-(5 / 2-\gamma) /(2-\gamma)+1}-T_{0}^{-(5 / 2-\gamma) /(2-\gamma)+1}}{T_{\max }^{-(5 / 2-\gamma) /(2-\gamma)+1}-T_{0}^{-(5 / 2-\gamma) /(2-\gamma)+1}} .
\end{gathered}
$$

We note that $T_{\max }$ may be complex if the planet never reaches $r=0$. The solution can easily be solved for $T$ for a given $M$, which can be converted to $t$ using the viscous disc expression from Eq. (B.5). The analytical expression for $T_{\max }$ is obtained by inserting $M=M_{\max }$ in Eq. (B.8).

If we assume for simplicity that $\dot{M}_{\mathrm{g}}$ is constant, then we can replace the complicated function of $T$ appearing on the righthand-side of Eq. (B.8) by $t-t_{0}$. In that case (setting $M_{0}=t_{0}=0$ also) we have for $t_{\max }$ the simple expression

$$
\begin{aligned}
t_{\max }= & r_{0}^{1-\zeta}(12 / 5) M_{\max }^{1 / 3} \\
& \times \frac{2 \pi(3 / 2)[(2 / 3)(S t / \alpha) \chi+1] \alpha c_{\mathrm{s} 1}^{2} \mathrm{AU}^{\zeta}}{2(S t / 0.1)^{2 / 3} G M_{\star}\left(3 M_{\star}\right)^{-2 / 3} \xi \dot{M}_{\mathrm{g} 0}} .
\end{aligned}
$$

The time-evolution of the growth track follows in the limit $M_{0}=t_{0}=0$ as $t / t_{\max }=(5 / 3)\left(M / M_{\max }\right)^{1 / 3}-(1 / 4)\left(M / M_{\max }\right)^{5 / 3}$. We can scale the timescale to migrate to the star, $t_{\max }$, to 
typical disc values,

$$
\begin{aligned}
t_{\max }= & 0.29 \mathrm{Myr}\left\{\frac{[(2 / 3)(S t / \alpha) \chi+1] / 2.9}{(S t / 0.01)^{2 / 3}}\right\}\left(\frac{\xi}{0.01}\right)^{-1} \\
& \times\left(\frac{M_{\star}}{M_{\odot}}\right)^{-1 / 3}\left(\frac{c_{\mathrm{s} 1}}{6.5 \times 10^{2} \mathrm{~m} \mathrm{~s}^{-1}}\right)^{2} \\
& \times\left(\frac{\alpha}{0.01}\right)\left(\frac{\dot{M}_{\mathrm{g} 0}}{10^{-7} M_{\odot} \mathrm{yr}^{-1}}\right)^{-1} \\
& \times\left(\frac{M_{\max }}{10 M_{\mathrm{E}}}\right)^{1 / 3}\left(\frac{r_{0}}{25 \mathrm{AU}}\right)^{1-\zeta}
\end{aligned}
$$

The relevant value of $M_{\max }$ for the given parameters can be inserted from Eq. (23). However, we note that $t_{\max }$ depends weakly on the maximum mass, as $M_{\max }^{1 / 3}$. Hence the parameter dependencies in Eq. (23) are much weaker than those explicit in Eq. (B.11) above. One can also approximately use $t_{\max }$ as the time to reach the isolation mass, as the isolation mass and the maximum mass are both reached after significant migration when $r_{0} \gg 10 \mathrm{AU}$. Under all circumstances $t_{\max }$ gives an upper limit to the time taken to reach the isolation mass. 